\title{
Adaptive Finite-Time Fault-Tolerant Control for Half-Vehicle Active Suspension Systems with Output Constraints and Random Actuator Failures
}

\author{
Jie $\operatorname{Lan}^{1,2}$ and Tongyu Xu $\mathbb{1}^{1}$ \\ ${ }^{1}$ College of Information and Electrical Engineering, Shenyang Agricultural University, Shenyang 110000, Liaoning, China \\ ${ }^{2}$ College of Science, Liaoning University of Technology, Jinzhou 121001, Liaoning, China \\ Correspondence should be addressed to Tongyu Xu; xutongyu@syau.edu.cn
}

Received 29 April 2021; Revised 4 June 2021; Accepted 21 June 2021; Published 10 July 2021

Academic Editor: Rui Wang

Copyright (c) 2021 Jie Lan and Tongyu Xu. This is an open access article distributed under the Creative Commons Attribution License, which permits unrestricted use, distribution, and reproduction in any medium, provided the original work is properly cited.

\begin{abstract}
The problem of adaptive finite-time fault-tolerant control (FTC) and output constraints for a class of uncertain nonlinear halfvehicle active suspension systems (ASSs) are investigated in this work. Markovian variables are used to denote in terms of different random actuators failures. In adaptive backstepping design procedure, barrier Lyapunov functions (BLFs) are adopted to constrain vertical motion and pitch motion to suppress the vibrations. Unknown functions and coefficients are approximated by the neural network (NN). Assisted by the stochastic practical finite-time theory and FTC theory, the proposed controller can ensure systems achieve stability in a finite time. Meanwhile, displacement and pitch angle in systems would not violate their maximum values, which imply both ride comfort and safety have been enhanced. In addition, all the signals in the closed-loop systems can be guaranteed to be semiglobal finite-time stable in probability (SGFSP). The simulation results illustrate the validity of the established scheme.
\end{abstract}

\section{Introduction}

With the development of modern industrial automation, vehicles play a more and more important role in people's production and life. Suspension as an important part, the damping effect to a great extent determines the comfort and safety of the automobile. Compared to the traditional passive and semiactive suspension systems, active suspension systems (ASSs) can provide better dynamic adjustment damping, potential road handling capacity, extreme ride comfort, and suspension deflection [1-3]. The design of complex mechanical engineering of ASSs had become a hot issue in the past two decades.

ASSs are often simplified into the full-vehicle model, halfvehicle model, and quarter-vehicle model. The actuators are parallel inserted to the components that provide external forces to increase or dissipate the energy of the ASSs and manage the tradeoffs between conflicting performance indicators. Many remarkable results were reported in [4-8] with the vertical motion of the quarter ASSs as the research topic. However, pitch motion was ignored that also directly affected the ride comfort and safety. Research on half-vehicle ASSs mainly focused on the discussions of pitch motion and vertical motion [9-17]. For ASSs, some inevitable uncertainties were in the design of controllers in [9-13], such as body mass, mass moment of inertia, and modeling uncertainties. But, these control methods [9-11] did not achieve good performance in estimating real valves. In $[12,13]$, they proposed adaptive control schemes by adding new leakage items to the update rules. In [14], the damping coefficient and spring stiffness of the tires were considered in the suspension as random uncertain parameters. The uncertain actuator was discussed and eliminated the influence by continuous-time homogeneous [15]. However, many progresses have been made for uncertain nonlinear ASSs, and a few studies were on the constraint of half-vehicle models. 
The actual mechanical systems need to keep the output or states within certain ranges; otherwise, the system performance would degrade. The properties of prescribed performance control [17] and barrier Lyapunov functions (BLFs) provide effective methods to deal with constraints, and a large number of results have been obtained for output constraints of various nonlinear systems and practical systems [18-26]. The asymmetric BLFs were coped with the position constraint problem of the marine vessel [24]. Due to the limitation of physical factors in suspension fields, it is worth noting that $[25,26]$ reported the output and timevarying output constraint of vertical motion for the quarter ASSs. However, for the half-vehicle ASSs, considering above output constraints had been carried out.

Theoretically, the aforementioned works can only guarantee the desired system performance as time approaches infinity. However, the actual mechanical control should achieve the expected transient system performance. The design of finite-time control for nonlinear systems has attracted considerable attention. The finite-time Lyapunov stability theorem was first proposed in [27]. Based on this theory, the continuous finite-time control for nonlinear systems was proposed in [28-34], robotic manipulators in [28], switched systems in [32-34], and Markovian jump systems in $[35,36]$. The concept of semiglobal practical finite-time stability (SGPFS) was proposed in different forms in [31-36]. The adaptive fuzzy finite-time control scheme of general uncertain nonlinear systems is discussed in [36]. Furthermore, Cai and Xiang expanded SGPFS to nonstrict nonlinear systems in [37]. Sui et al. expanded SGPFS for nontriangular stochastic nonlinear systems in [38]. The finite-time results of nonlinear quarter ASSs had been made in $[39,40]$. The finite-time results of nonlinear half ASSs had been made in [41], but there was no constraint study on the individual states. For nonlinear strict feedback systems, both output constraint and finite-time control design had been completed in [42, 43]. However, for uncertain nonlinear half ASSs, there are few results on how to implement finite-time control associated with output constraints.

On the other hand, actuator failures are inevitable due to the influence of external environment, mechanical system failures, operation errors, and human factors in practical systems. These faults can seriously have an impact on system stability, degradation, and even catastrophic risks. Most of the above studies assumed that all actuators or sensors were in normal operation. Fault-tolerant control (FTC) strategies can compensate the faults and maintain acceptable system performance. Many methods to deal with actuator failures, such as the pseudoinverse method was in [44], model prediction method was in [45], and sliding control was in [46], by applying the adaptive backstepping techniques for linear systems in [47] and nonlinear strict feedback systems in [48-50]. Failures should be random. The actuator of states can switch between various modes in a random way. Given enough historical data, the states of the actuator can be modeled as Markov states in [51]. In the process, the failures of different actuators also meet the requirements of different Markov processes. Each actuator is independent and can fail at any sampling time. In [52], an adaptive fault compensation for a class of nonlinear uncertain systems with random actuator faults was studied and a random function to scale actuator faults by Markov correlation variables was proposed. In [53], random faults between different actuators in the half ASSs were considered for the first time. Motivated by the above observations and existing research results, this study proposes an adaptive NN finite-time FTC scheme for uncertain nonlinear half-vehicle ASSs with output constraints. The three main advantages of the proposed scheme can be listed as follows:

(1) Compared with existing adaptive FTC studies, the problem for half ASSs subject to infinite stochastic actuator failures and the states of multiactuators modeled by different Markovian processes has not received enough attention. Particularly, considering finite-time control, the additional correlation terms generated by the infinitesimal generator are handled by the stochastic finite-time control theorem.

(2) In comparison with existing constraints, it is asymmetry, which can restrain different outputs of displace and pitch angle more reasonable and reduce the vibration in uncertain nonlinear half ASSs. Moreover, the finite-time FTC control strategy can also enable the practical control systems to realize the transient system stability.

(3) In comparison with existing adaptive finite-time control, it considers a class of uncertain nonlinear half-ASSs subject to stochastic actuator failures. It should dispose random terms which makes the existing stability criteria in [53-55] are invalid. Concurrently, the asymmetric output constraints for different factors have been considered. The Lyapunov is proved SGPFS.

This work is organized as follows. In Section 2, the half active suspension systems and control objectives are shown. Section 3 presents the design procedures of the adaptive finite-time fault-tolerant controller designed based on stochastic Lyapunov function and zero dynamic. In Section 4, an example to show that the constructed method is effective. In Section 5, it demonstrates a conclusion about the results of this work and future work.

\section{System Description and Preliminaries}

2.1. Nonlinear Half-Vehicle Suspension Systems. Figure 1 shows a half-vehicle suspension model. $M$ represents the mass of the vehicle body. $I$ is the mass moment of inertia. $m_{f}$ and $m_{r}$ are the defined masses of front and rear wheels, respectively. $D_{c}$ stands for the vertical displacement of the vehicle body. $\varphi$ represents the pitch angle. $D_{f}$ and $D_{r}$ stand for the displacements of the front and rear vehicle body, respectively. $D_{1}$ and $D_{2}$ are the displacements of the front and rear wheels, respectively. $D_{01}$ and $D_{02}$ represent the road inputs of corresponding wheels. $F_{\mathrm{sr}}, F_{\mathrm{tr}}, F_{\mathrm{sf}}$, and $F_{\mathrm{tf}}$ are defined as the forces produced by the related stiffness. $F_{\mathrm{dr}}$, $F_{\mathrm{br}}, F_{\mathrm{df}}$, and $F_{\mathrm{bf}}$ are defined as the forces produced by the related dampers. $u_{f}$ and $u_{r}$ represent the control forces of the front and rear ASSs. 


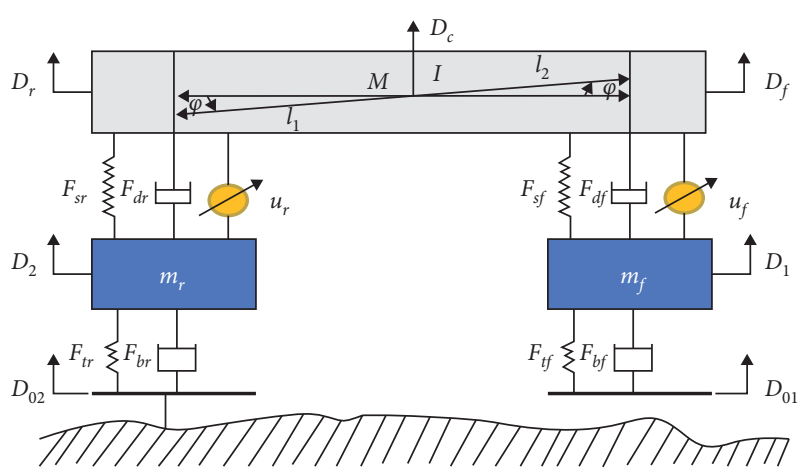

Figure 1: The nonlinear half-vehicle suspension model.

For half ASSs, the state space equations of vertical motion and pitch motion are shown in the following equation:

$$
\left\{\begin{array}{l}
M \ddot{D}_{c}+F_{\mathrm{sf}}+F_{\mathrm{df}}+F_{\mathrm{sr}}+F_{\mathrm{dr}}-u_{z}=0, \\
I \ddot{\varphi}+l_{2}\left(F_{\mathrm{sf}}+F_{\mathrm{df}}\right)-l_{1}\left(F_{\mathrm{sr}}+F_{\mathrm{dr}}\right)-u_{\varphi}=0, \\
m_{f} \ddot{D}_{1}-F_{\mathrm{sf}}-F_{\mathrm{df}}+F_{\mathrm{tf}}+F_{\mathrm{bf}}+u_{f}=0, \\
m_{r} \ddot{D}_{2}-F_{\mathrm{sf}}-F_{\mathrm{dr}}+F_{\mathrm{tr}}+F_{\mathrm{br}}+u_{r}=0,
\end{array}\right.
$$

where $u_{z}=u_{r}+u_{f}$ and $u_{\varphi}=l_{2} u_{f}-l_{1} u_{r}$.

The stochastic actuator failures are considered and described as follows:

$$
\begin{aligned}
& u_{f}(t)=g_{1}\left(r_{1}(t)\right) \Psi_{f}(t), \\
& u_{r}(t)=g_{2}\left(r_{2}(t)\right) \Psi_{r}(t),
\end{aligned}
$$

where $\Psi_{f}(t)$ and $\Psi_{r}(t)$ are the inputs of the front actuator and rear actuator, respectively. $r_{1}(t)$ and $r_{2}(t)$ are the independent irreducible right continuous homogeneous Markovian processes on the probability space $(\Omega, E, P)$, taking values in a finite set $S=\{1,2, \ldots, N\}$ with generator matrix $\Pi=\left(\Upsilon_{p q}\right)_{N \times N}$, where $\Upsilon_{p q}>0$ is the transition rate from mode $p$ to mode $q$ if $p \neq q$, and $\Upsilon_{p p}=\sum_{q=1, p \neq q}^{N} \Upsilon_{p q}$.

In addition, $g_{1}\left(r_{1}(t)\right)$ and $g_{2}\left(r_{2}(t)\right)$ are the stochastic functions which represent the failure scaling factors for two different actuators and take values on the interval $\left[g_{1 \min }, 1\right]\left[g_{2 \min }, 1\right]$, and $g_{1 \min }>0, g_{2 \min }>0$.

Remark 1. The values $g_{1}\left(r_{1}(t)\right)$ and $g_{2}\left(r_{2}(t)\right)$ meet as follows,

(1) When $g_{1}\left(r_{1}(t)\right)=1$ or $g_{2}\left(r_{2}(t)\right)=1$, the front actuator or the rear actuator is healthy

(2) When $g_{1}\left(r_{1}(t)\right) \in\left[g_{1 \mathrm{~min}}, 1\right)$ or $g_{2}\left(r_{2}(t)\right) \in\left[g_{2 \min }, 1\right)$, there is partial failure of the corresponding actuator

Each actuator will switch randomly between above two states. The problem of FTC considered in the systems was assumed that once the actuator failed, it would keep the fault state for the rest operation. However, the failure may be intermittent, and the actuator may repetitiously fail with different failure modes. Meanwhile, the modes, times, and patterns of actuator failures are essentially random. Then, actuator failures in (2) are more complicated and practical.

In order to facilitate the design and analysis of the adaptive finite-time fault-tolerant control method, the state variables need to be defined as follows:

$$
\begin{aligned}
& x_{1}=D_{c} \text {, } \\
& x_{2}=\dot{D}_{c} \text {, } \\
& x_{3}=\varphi \text {, } \\
& x_{4}=\dot{\varphi} \text {, } \\
& x_{5}=D_{1} \text {, } \\
& x_{6}=\dot{D}_{1} \text {, } \\
& x_{7}=D_{2} \text {, } \\
& x_{8}=\dot{D}_{2} \text {, } \\
& x=\left[\begin{array}{llllllll}
x_{1} & x_{2} & x_{3} & x_{4} & x_{5} & x_{6} & x_{7} & x_{8}
\end{array}\right]^{T} .
\end{aligned}
$$

Then, it obtains

$$
\left\{\begin{array}{l}
\dot{x}_{1}=x_{2}, \\
\dot{x}_{2}=F_{1}+\rho_{1}\left(g_{1}\left(r_{1}(t)\right) \Psi_{f}(t)+g_{2}\left(r_{2}(t)\right) \Psi_{r}(t)\right), \\
\dot{x}_{3}=x_{4}, \\
\dot{x}_{4}=F_{2}+\rho_{2}\left(l_{2} g_{1}\left(r_{1}(t)\right) \Psi_{f}(t)-l_{1} g_{2}\left(r_{2}(t)\right) \Psi_{r}(t)\right),
\end{array}\right.
$$

$$
\left\{\begin{array}{l}
\dot{x}_{5}=x_{6}, \\
\dot{x}_{6}=\frac{1}{m_{f}}\left(F_{\mathrm{sf}}+F_{\mathrm{df}}-F_{\mathrm{tf}}-F_{\mathrm{bf}}-g_{1}\left(r_{1}(t)\right) \Psi_{f}(t)\right), \\
\dot{x}_{7}=x_{8}, \\
\dot{x}_{8}=\frac{1}{m_{r}}\left(F_{\mathrm{sr}}+F_{\mathrm{dr}}-F_{\mathrm{tr}}-F_{\mathrm{br}}-g_{2}\left(r_{2}(t)\right) \Psi_{r}(t)\right),
\end{array}\right.
$$

where $\rho_{1}=1 / M, \rho_{2}=1 / I, F_{1}=\rho_{1}\left(-F_{\mathrm{sf}}-F_{\mathrm{df}}-F_{\mathrm{sr}}-F_{\mathrm{dr}}\right)$, and $F_{2}=\rho_{2}\left(-l_{2}\left(F_{\mathrm{sf}}+F_{\mathrm{df}}\right)+l_{1}\left(F_{\mathrm{sr}}+F_{\mathrm{dr}}\right)\right)$.

The mass $M$ and the mass moment of inertia $I$ are uncertain due to the uncertainties of passengers and the load quality of cargoes. Therefore, $F_{1}$ and $F_{2}$ are the uncertainty and unknown functions.

2.2. Control Objectives. For different types of vehicles, improving ride comfort and safety is one of the most important requirements. Due to the hardware limitations, the following three requirements must be considered in the design and control process of ASSs.

First, the controllers $u_{f}$ and $u_{r}$ are subjected to random Markovian jumping failures in (2). The vertical displacement $D_{c}$ and pitch angle motion $\varphi$ are considered and limited in safety ranges. $D_{c}$ and $\varphi$ should be smaller which can largely enhance ride comfort. 
Second, considering the driving safety, the wheels should make uninterrupted contact with the ground. It means that the dynamic tire load must be less than the static load, i.e.,

$$
\begin{aligned}
& \left|F_{\mathrm{tf}}+F_{\mathrm{bf}}\right| \leq F_{f}, \\
& \left|F_{\mathrm{tr}}+F_{\mathrm{br}}\right| \leq F_{r},
\end{aligned}
$$

where $F_{f}+F_{r}=\left(M+m_{f}+m_{r}\right) g$, and $F_{f}\left(l_{1}+l_{2}\right)=$ $m_{f} g\left(l_{1}+l_{2}\right)+M l_{1} g$.

Last, suspension constraints must be guaranteed because of the confined mechanical space, that is, suspension deflections should not exceed their maximum values.

$$
\begin{aligned}
& \left|\Delta D_{f}\right| \leq \Delta \bar{D}_{f}, \\
& \left|\Delta D_{r}\right| \leq \Delta \bar{D}_{r},
\end{aligned}
$$

where $\Delta D_{f}=D_{c}+l_{2} \sin \varphi-D_{1}, \quad$ and $\quad \Delta D_{r}=D_{c}-l_{1}$ $\sin \varphi-D_{2}$.

There are some assumptions, definitions, and lemmas presented in order to facilitate the design and analysis of adaptive control scheme.

Assumption 1. For given constants $\underline{k}_{c 1}>0, \bar{k}_{c 1}>0, \underline{k}_{c 2}>0$, and $\bar{k}_{c 2}>0$, the following results on $y_{1 d}, y_{3 d}, \dot{y}_{1 d}$, and $\dot{y}_{3 d}$ hold, i.e.,

$$
\begin{aligned}
-\underline{B}_{11} \leq y_{1 d} & \leq \bar{B}_{11}, \\
\left|\dot{y}_{1 d}\right| & \leq \bar{B}_{12}, \\
-\underline{B}_{31} \leq y_{3 d} & \leq \bar{B}_{31}, \\
\left|\dot{y}_{3 d}\right| & \leq \bar{B}_{32},
\end{aligned}
$$

where $\bar{B}_{11}<\bar{k}_{c 1}, \underline{B}_{11} \leq \underline{k}_{c 1}, \bar{B}_{31}<\bar{k}_{c 2}$, and $\underline{B}_{31} \leq \underline{k}_{c 2}$, for positive parameters $\underline{B}_{11}, \bar{B}_{11}, \underline{B}_{31}, \bar{B}_{31}, \bar{B}_{12}$, and $\bar{B}_{32}$ are selected and positive parameters.

Assumption 2. In general, the inputs of the road and their time derivative of $D_{01}$ and $D_{02}$ are limited. Therefore, existing positive parameters make sure the following inequality:

$$
\begin{aligned}
& \left|D_{01}(t)\right| \leq \bar{D}_{11}, \\
& \left|\dot{D}_{01}(t)\right| \leq \bar{D}_{12}, \\
& \left|D_{02}(t)\right| \leq \bar{D}_{21}, \\
& \left|\dot{D}_{02}(t)\right| \leq \bar{D}_{22},
\end{aligned}
$$

where the parameters $\bar{D}_{11}, \bar{D}_{12}, \bar{D}_{21}$, and $\bar{D}_{22}$ are positive.

Definition 1 (see [35]). Consider the following nonlinear systems:

$$
\begin{aligned}
\dot{\zeta} & =g(\zeta, u), \\
g(0,0) & =0, \quad \zeta \in \Re^{n}, u \in \Re^{n},
\end{aligned}
$$

where $\zeta$ and $u$ represent the state and input vectors, respectively. If any initial condition $\zeta\left(t_{0}\right)$ satisfies $\zeta\left(t_{0}\right)=\zeta_{0}$, for $t \geq t_{0}+T$, the system (10) is semiglobal practical finitetime stable (SGPFS). In addition, the state vector $\zeta(t)$ satisfies $\|\zeta(t)\| \leq \bar{\zeta}$, for $t \geq t_{0}+T$, where $\bar{\zeta}$ is a positive parameter and a deposit time $T\left(\zeta_{0}, \bar{\zeta}\right)$ satisfies $0<T\left(\zeta_{0}, \bar{\zeta}\right)<\infty$.

Lemma 1 (see [36]). For any $\gamma_{i} \in R, i=1,2, \ldots, n, 0<a<1$, $0<b<2$, the following inequality can be constructed, namely,

$$
\begin{aligned}
& \left(\left|\gamma_{1}\right|+\left|\gamma_{2}\right|+\cdots+\left|\gamma_{n}\right|\right)^{a} \leq\left|\gamma_{1}\right|^{a}+\left|\gamma_{2}\right|^{a}+\cdots+\left|\gamma_{n}\right|^{a}, \\
& \left(\left|\gamma_{1}\right|^{2}+\left|\gamma_{2}\right|^{2}+\cdots+\left|\gamma_{n}\right|^{2}\right)^{b} \leq\left(\left|\gamma_{1}\right|^{a}+\left|\gamma_{2}\right|^{a}+\cdots+\left|\gamma_{n}\right|^{a}\right)^{2} .
\end{aligned}
$$

Lemma 2 (see [37]). Consider the Lyapunov function $V(\zeta)$ with stochastic terms, and the following inequality holds for a nonlinear system (10), namely,

$$
L V(\zeta) \leq-\ell V^{a}(\zeta)+\hbar
$$

where $L$ is the differential operator, the constants $\ell$ and $\hbar$ are positive, and $0<a<1 . \zeta \in R^{n}$ and $t \geq t_{0}$. Then, stochastic trajectory of (10) is SGPFS.

Lemma 3 (see [40]). For any $z$ and $\alpha$ are real variables, the following inequality can be constructed, namely,

$$
|z|^{a}|\alpha|^{b} \leq \frac{a}{a+b} \mu|z|^{a+b}+\frac{b}{a+b} \mu^{-(a / b)}|\alpha|^{a+b},
$$

where the parameters $a, b$, and $\mu$ are positive.

Lemma 4 (see [23]). For all $\left|\gamma_{1}\right| \leq k_{b 1}$, the following inequality can be constructed as

$$
\log \frac{1}{k_{b 1}^{2}-\gamma_{1}^{2}} \leq \frac{\gamma_{1}^{2}}{k_{b 1}^{2}-\gamma_{1}^{2}}
$$

\section{Adaptive Finite-Time Fault-Tolerant Controller Design Based on Stochastic Lyapunov Function and Zero Dynamic}

For a class of uncertain nonlinear half ASSs, the universal approximation property of NN solves the uncertainty, and then, the outputs of vertical motion and pitch angle motion are constrained by using asymmetrical log-BLFs. The adaptive backstepping technique is used to address the functions with random generality variables generated by the infinitesimal generator due to random actuator failures. The Lyapunov stability is proved by the SGPFS and zero dynamic theorem.

In order to facilitate the design of controllers, there are some given coordinate transformations, i.e.,

$$
\begin{aligned}
& \gamma_{1}=x_{1}-y_{1 d}, \\
& \gamma_{2}=x_{2}-\beta_{1}, \\
& \gamma_{3}=x_{3}-y_{3 d}, \\
& \gamma_{4}=x_{4}-\beta_{3},
\end{aligned}
$$


where $\beta_{1}$ and $\beta_{3}$ are the virtual control signals. $\gamma_{i}$ for $1 \leq i \leq 4$ is the error variable. $y_{1 d}$ and $y_{3 d}$ are the desired vertical displacement and pitch angle, respectively.

\subsection{Finite-Time Constraint Control Scheme Design for Vertical} Motion and Pitch Angle Motion. Now, for the vertical motion of active suspension systems, more details on finite-time approach will be given in the next section.

Step 1. Select $V=V\left(x(t), r_{1}(t), r_{2}(t)\right)$ as a Lyapunov function candidate in the following form:

$$
\begin{aligned}
V= & \sum_{i=1,3}\left[\frac{1-q\left(\gamma_{i}\right)}{2} \log \frac{k_{a i}^{2}}{k_{a i}^{2}-\gamma_{i}^{2}}+\frac{q\left(\gamma_{i}\right)}{2} \log \frac{k_{b i}^{2}}{k_{b i}^{2}-\gamma_{i}^{2}}\right] \\
& +\sum_{i=1}^{2}\left(\frac{1}{2 \rho_{i}} \gamma_{2 i}^{2}+\frac{1}{2 \eta_{i}} \tilde{\theta}_{i}^{2}+\frac{1}{2 \chi_{i}} \tilde{g}_{i}^{2}\left(r_{i}(t)\right)\right),
\end{aligned}
$$

where $\quad x(t)=\left(x_{1}(t), x_{2}(t), x_{3}(t), x_{4}(t)\right), \quad \gamma_{1}=\left(x_{1}, r_{1}(t)\right.$, $\left.r_{2}(t)\right), \gamma_{2}=\left(x_{1}, x_{2}, r_{1}(t), r_{2}(t)\right), \gamma_{3}=\left(x_{3}, r_{1}(t), r_{2}(t)\right)$, and $\gamma_{4}=\left(x_{3}, x_{4}, r_{1}(t), r_{2}(t)\right)$. The parameters $\eta_{i}$ and $\chi_{i}$ are positive, and $\tilde{\theta}_{i}$ and $\widetilde{g}_{i}\left(r_{i}(t)\right)$ represent the errors. $\widehat{\theta}_{i}$ is the estimator of $\theta_{i} \cdot \hat{g}_{i}\left(r_{i}(t)\right)$ is the estimator of $g_{i}\left(r_{i}(t)\right)$. Then, $\widetilde{\theta}_{i}=\theta_{i}-\widehat{\theta}_{i}, \quad \widetilde{g}_{i}\left(r_{i}(t)\right)=g_{i}\left(r_{i}(t)\right)-\widehat{g}_{i}\left(r_{i}(t)\right), \quad i=1,2$. It would make an assumption: $\forall t>0, r_{1}(t)=p_{1}, r_{2}(t)=p_{2}$, and $p_{1}, p_{2} \in S$.

Remark 2. For quarter ASSs, the study in [30] only showed the vehicle body's displacement constraint method by the symmetric BLFs. In contrast, we further study the pitch angle constraint of nonlinear uncertain half ASSs by asymmetric BLFs in this study.

In addition, the virtual controller signal is designed as

$$
\beta_{i}=-\frac{c_{i} \gamma_{i}}{2}\left(\frac{1-q\left(\gamma_{i}\right)}{k_{a i}^{2}-\gamma_{i}^{2}}+\frac{q\left(\gamma_{i}\right)}{k_{b i}^{2}-\gamma_{i}^{2}}\right)+\dot{y}_{i d},
$$

where $i=1,3$. The parameter $c_{i}$ is a design parameter, $c_{i}>0$. $k_{a i}$ and $k_{b i}$ are the constraint bounded of corresponding variables, respectively. Moreover,

$$
q(\cdot)= \begin{cases}1, & \cdot \geq 0 \\ 0, & \cdot<0\end{cases}
$$

Then, according to the infinitesimal generator of $V$, it gets

$$
\begin{aligned}
\mathrm{LV}= & \sum_{i=1,3}\left[\left(1-q\left(\gamma_{i}\right)\right) \frac{\gamma_{i} \dot{\gamma}_{i}}{k_{a i}^{2}-\gamma_{i}^{2}}+q\left(\gamma_{i}\right) \frac{\gamma_{i} \dot{\gamma}_{i}}{k_{b i}^{2}-\gamma_{i}^{2}}\right] \\
& +\sum_{i=1}^{2}\left(\frac{1}{\rho_{i}} \gamma_{2 i} \dot{\gamma}_{2 i}+\frac{1}{\eta_{i}} \tilde{\theta}_{i} \dot{\tilde{\theta}}_{i}+\frac{1}{\chi_{i}} \widetilde{g}_{i}\left(p_{i}\right) \dot{\tilde{g}}_{i}\left(p_{i}\right)\right) \\
& +\sum_{q_{1}=1}^{N} \Upsilon_{p_{1} q_{1}} V_{1}+\sum_{q_{2}=1}^{N} \Upsilon_{p_{2} q_{2}} V_{2},
\end{aligned}
$$

where $V_{1}=\left(x(t), p_{2}, q_{1}\right), V_{2}=\left(x(t), p_{1}, q_{2}\right)$, and $\Upsilon_{p_{i} q_{i}}>0$ is the transition rate, $i=1,2$.

Remark 3. $\sum_{q_{1}=1}^{N} \Upsilon_{p_{1} q_{1}} V_{1}$ and $\sum_{q_{2}=1}^{N} \Upsilon_{p_{2} q_{2}} V_{2}$ are the additional terms due to the involvement of Markovian variables $r_{1}(t)$ and $r_{2}(t)$, which do not exist in the determined situation. The extratransition rate-related terms appear in the infinitesimal generator of Lyapunov candidate function. These additions need further processing. It is a challenge that cannot be ignored in stability analysis.

From the previous definition, $i=1,2$, there are

$$
\begin{array}{r}
\dot{\vec{g}}_{i}\left(r_{i}(t)\right)=-\dot{\hat{g}}_{i}\left(r_{i}(t)\right), \\
\dot{\tilde{\theta}}_{i}=-\dot{\hat{\theta}}_{i} .
\end{array}
$$

Combining with (19) and (20) can obtain

$$
\begin{aligned}
\mathrm{LV}= & \sum_{i=1,3} \gamma_{i}\left[\frac{1-q\left(\gamma_{i}\right)}{k_{a i}^{2}-\gamma_{i}^{2}}+\frac{q\left(\gamma_{i}\right)}{k_{b i}^{2}-\gamma_{i}^{2}}\right] \dot{\gamma}_{i}+\sum_{i=1}^{2} \frac{1}{\rho_{i}} \gamma_{2 i} \dot{\gamma}_{2 i} \\
& -\sum_{i=1}^{2}\left(\frac{1}{\chi_{i}} \tilde{g}_{i}\left(p_{i}\right) \dot{\hat{g}}_{i}\left(p_{i}\right)+\frac{1}{\eta_{i}} \tilde{\theta}_{i} \dot{\hat{\theta}}_{i}\right) \\
& +\sum_{q_{1}=1}^{N} \Upsilon_{p_{1} q_{1}} V_{1}+\sum_{q_{2}=1}^{N} \Upsilon_{p_{2} q_{2}} V_{2} .
\end{aligned}
$$

Furthermore, taking the derivative of $\gamma_{i}$, it yields

$$
\dot{\gamma}_{i}=\gamma_{i+1}+\beta_{i}-\dot{y}_{i d}, \quad i=1,3 \text {. }
$$

Substituting (17) and (22) into (21), it can attain

$$
\begin{aligned}
\gamma_{i}\left[\frac{1-q\left(\gamma_{i}\right)}{k_{a i}^{2}-\gamma_{i}^{2}}+\frac{q\left(\gamma_{i}\right)}{k_{b i}^{2}-\gamma_{i}^{2}}\right] \dot{\gamma}_{i} & =\lambda_{i}\left(\gamma_{i} \gamma_{i+1}-\frac{c_{i} \gamma_{i}^{2}}{2}\left(\frac{1-q\left(\gamma_{i}\right)}{k_{a i}^{2}-\gamma_{i}^{2}}+\frac{q\left(\gamma_{i}\right)}{k_{b i}^{2}-\gamma_{i}^{2}}\right)\right) \\
& =\lambda_{i} \gamma_{i} \gamma_{i+1}-c_{i} \frac{1-q\left(\gamma_{i}\right)}{2} \frac{\gamma_{i}^{2}}{k_{a i}^{2}-\gamma_{i}^{2}}-c_{i} \frac{q\left(\gamma_{i}\right)}{2} \frac{\gamma_{i}^{2}}{k_{b i}^{2}-\gamma_{i}^{2}}
\end{aligned}
$$

where $\lambda_{i}=\left(\left(1-q\left(\gamma_{i}\right)\right) / k_{a i}^{2}-\gamma_{i}^{2}\right)+\left(q\left(\gamma_{i}\right) /\left(k_{b i}^{2}-\gamma_{i}^{2}\right)\right), c_{i}$ is a positive design parameter, and $i=1,3$.
Based Lemma 3, let $z_{i}=\left(\gamma_{i}^{2} / 2\left(k_{a i}^{2}-\gamma_{i}^{2}\right)\right), \alpha=1, a=$ $((2 n-1) / 2 n+1), n \in \mathbb{N}, b=1-a$, and $\mu=a^{(a /(1-a))}$ obtain 


$$
\left(\frac{\gamma_{i}^{2}}{2\left(k_{a i}^{2}-\gamma_{i}^{2}\right)}\right)^{a} \leq(1-a) \mu+\frac{\gamma_{i}^{2}}{2\left(k_{a i}^{2}-\gamma_{i}^{2}\right)} .
$$

Therefore, it gets

$$
-\frac{\gamma_{i}^{2}}{2\left(k_{a i}^{2}-\gamma_{i}^{2}\right)} \leq(1-a) \mu-\left(\frac{\gamma_{i}^{2}}{2\left(k_{a i}^{2}-\gamma_{i}^{2}\right)}\right)^{a} .
$$

Constructed in the same way, it obtains

$$
-\frac{\left(1-q\left(\gamma_{i}\right)\right) \gamma_{i}^{2}}{2\left(k_{a i}^{2}-\gamma_{i}^{2}\right)} \leq(1-a) \mu-\left(1-q\left(\gamma_{i}\right)\right)^{a}\left(\frac{\gamma_{i}^{2}}{2\left(k_{a i}^{2}-\gamma_{i}^{2}\right)}\right)^{a} \text {. }
$$

Similarly, it yields

$$
\begin{aligned}
& -\frac{\gamma_{i}^{2}}{2\left(k_{b i}^{2}-\gamma_{i}^{2}\right)} \leq(1-a) \mu-\left(\frac{\gamma_{i}^{2}}{2\left(k_{b i}^{2}-\gamma_{i}^{2}\right)}\right)^{a}, \\
& -\frac{q\left(\gamma_{i}\right) \gamma_{i}^{2}}{2\left(k_{b i}^{2}-\gamma_{i}^{2}\right)} \leq(1-a)-\left(q\left(\gamma_{i}\right)\right)^{a}\left(\frac{\gamma_{i}^{2}}{2\left(k_{b i}^{2}-\gamma_{i}^{2}\right)}\right)^{a} .
\end{aligned}
$$

According to Lemma 4, for any constant $a, 0<a<1$, the following inequality holds:

$$
\begin{aligned}
& \left(\log \frac{1}{k_{a i}^{2}-\gamma_{i}^{2}}\right)^{a} \leq\left(\frac{\gamma_{i}^{2}}{k_{a i}^{2}-\gamma_{i}^{2}}\right)^{a}, \\
& \left(\log \frac{1}{k_{b i}^{2}-\gamma_{i}^{2}}\right)^{a} \leq\left(\frac{\gamma_{i}^{2}}{k_{b i}^{2}-\gamma_{i}^{2}}\right)^{a} .
\end{aligned}
$$

Next, substituting (26)-(28) into (23), LV is expressed as

$$
\begin{aligned}
\mathrm{LV}= & \sum_{i=1,3}\left[\lambda_{i} \gamma_{i} \gamma_{i+1}-c_{i}\left(\frac{1-q\left(\gamma_{i}\right)}{2} \log \frac{1}{k_{a i}^{2}-\gamma_{i}^{2}}\right)^{a}\right. \\
& -c_{i}\left(\frac{q\left(\gamma_{i}\right)}{2} \log \frac{1}{k_{b i}^{2}-\gamma_{i}^{2}}\right)^{a}+\sum_{q_{1}=1}^{N} \Upsilon_{p_{1} q_{1}} V_{1}+\sum_{q_{2}=1}^{N} \Upsilon_{p_{2} q_{2}} V_{2}+\hbar_{1} \\
& +\sum_{i=1}^{2}\left(\frac{1}{\rho_{i}} \gamma_{2 i} \dot{\gamma}_{2 i}-\frac{1}{\chi_{i}} \widetilde{g}_{i}\left(p_{i}\right) \dot{\hat{g}}_{i}\left(p_{i}\right)-\frac{1}{\eta_{i}} \tilde{\theta}_{i} \dot{\hat{\theta}}_{i}\right),
\end{aligned}
$$

where $\hbar_{1}=\sum_{i=1,3} 2 c_{i}(1-a) \mu$.

Taking the derivative of $\gamma_{2}$ and $\gamma_{4}$, they yield

$$
\begin{aligned}
& \dot{\gamma}_{2}=F_{1}+\rho_{1} u_{z}-\dot{\beta}_{1}, \\
& \dot{\gamma}_{4}=F_{2}+\rho_{2} u_{\varphi}-\dot{\beta}_{3},
\end{aligned}
$$

where $\quad \dot{\beta}_{i}=\left(\partial \beta_{i} / \partial x_{i}\right) \dot{x}_{i}+\left(\partial \beta_{i} / \partial y_{i d}\right) \dot{y}_{i d}+\left(\partial \beta_{i} / \partial \dot{y}_{i d}\right) \ddot{y}_{i d}$, $i=1,3$. $\beta_{i}$ has been designed in (17). Then, $\sum_{i=1}^{2}\left(1 / \rho_{i}\right) \gamma_{2 i} \dot{\gamma}_{2 i}$ gets

$$
\begin{aligned}
& \frac{1}{\rho_{1}} \gamma_{2} \dot{\gamma}_{2}=\gamma_{2}\left(\bar{F}_{1}+u_{z}\right), \\
& \frac{1}{\rho_{2}} \gamma_{4} \dot{\gamma}_{4}=\gamma_{4}\left(\bar{F}_{2}+u_{\varphi}\right),
\end{aligned}
$$

where the unknown functions $\bar{F}_{1}$ and $\bar{F}_{2}$ are denoted as

$$
\begin{aligned}
& \bar{F}_{1}=\rho_{1}^{-1} F_{1}-\rho_{1}^{-1} \dot{\beta}_{1}, \\
& \bar{F}_{2}=\rho_{2}^{-1} F_{2}-\rho_{2}^{-1} \dot{\beta}_{3} .
\end{aligned}
$$

Using the powerful approximating ability of $\mathrm{NNs}$ in $[42,43]$ for uncertain nonlinear systems, $\bar{F}_{1}$ and $\bar{F}_{2}$ can employ

$$
\begin{aligned}
& \bar{F}_{1}=\Theta_{1} \Phi\left(Z_{1}\right)^{T}+\tau_{1}, \\
& \bar{F}_{2}=\Theta_{2} \Phi\left(Z_{2}\right)^{T}+\tau_{2},
\end{aligned}
$$

where $Z_{1}=\left[x_{1}, x_{2}, x_{3}, x_{4}, x_{5}, x_{6}, x_{7}, x_{8}, \beta_{1}, \dot{\beta}_{1}, y_{1 d}, \dot{y}_{1 d}\right]^{T}$, $Z_{2}=\left[x_{1}, x_{2}, x_{3}, x_{4}, x_{5}, x_{6}, x_{7}, x_{8}, \beta_{3}, \dot{\beta}_{3}, y_{3 d}, \dot{y}_{3 d}\right]^{T}$, and $\tau_{i}$ is the approximation error and the designed positive constant $\bar{\tau}_{i}$ that satisfies $\left|\tau_{i}\right| \leq \bar{\tau}_{i}$. More remarkable, $0<\Theta_{1 i} \Phi\left(Z_{1}\right)<1$ and $0<\Theta_{2 i} \Phi\left(Z_{2}\right)<1$. Furthermore, we can draw a conclusion $\quad 0<\Phi_{1}^{T}\left(Z_{1}\right) \Phi_{1}\left(Z_{1}\right) \leq N_{1} \quad$ and $\quad 0<\Phi_{2}^{T}\left(Z_{2}\right) \Phi_{2}$ $\left(Z_{2}\right) \leq N_{2}$, where $N_{1}$ and $N_{2}$ are the corresponding numbers of NNs nodes.

Then, applying Young's inequality, it can get the following:

$$
\begin{aligned}
\gamma_{2 i} \Theta_{i} \Phi\left(Z_{i}\right)^{T} & \leq \frac{\gamma_{2 i}^{2}\left\|\Theta_{i}\right\|^{2} \Phi\left(Z_{i}\right)^{T} \Phi\left(Z_{i}\right)}{2 \delta_{i}^{2}}+\frac{\delta_{i}^{2}}{2} \\
& =\frac{\gamma_{2 i}^{2} \theta_{i}}{2 \delta_{i}^{2}}+\frac{\delta_{i}^{2}}{2}, \\
\gamma_{2 i} \tau_{i} & \leq \frac{1}{2} \gamma_{2 i}^{2}+\frac{1}{2} \tau_{i}^{2} \leq \frac{1}{2} \gamma_{2 i}^{2}+\frac{1}{2} \bar{\tau}_{i}^{2},
\end{aligned}
$$

where $\theta_{i}=N_{i}\left\|\Theta_{i}\right\|^{2}$ and the design parameter $\delta_{i}$ is positive.

Substituting (33)-(35) into (31), it obtains

$$
\begin{aligned}
\sum_{i=1}^{2} \frac{1}{\rho_{i}} \gamma_{2 i} \dot{\gamma}_{2 i} \leq & \gamma_{2}\left(\frac{\gamma_{2} \theta_{1}}{2 \delta_{1}^{2}}+\frac{\gamma_{2}}{2}+u_{z}\right)+\gamma_{4}\left(\frac{\gamma_{4} \theta_{2}}{2 \delta_{2}^{2}}+\frac{\gamma_{4}}{2}+u_{\varphi}\right) \\
& +\sum_{i=1}^{2}\left(\frac{\delta_{i}^{2}}{2}+\frac{\bar{\tau}_{i}^{2}}{2}\right) .
\end{aligned}
$$

Substituting (36) into (29), it can obtain

$$
\begin{aligned}
\mathrm{LV}= & \sum_{i=1,3}\left[\lambda_{i} \gamma_{i} \gamma_{i+1}-c_{i}\left(\frac{1-q\left(\gamma_{i}\right)}{2} \log \frac{1}{k_{a i}^{2}-\gamma_{i}^{2}}\right)^{a}\right. \\
& -c_{i}\left(\frac{q\left(\gamma_{i}\right)}{2} \log \frac{1}{k_{b i}^{2}-\gamma_{i}^{2}}\right)^{a}+\gamma_{2}\left(\frac{\gamma_{2} \theta_{1}}{2 \delta_{1}^{2}}+\frac{\gamma_{2}}{2}+u_{z}\right) \\
& +\gamma_{4}\left(\frac{\gamma_{4} \theta_{2}}{2 \delta_{2}^{2}}+\frac{\gamma_{4}}{2}+u_{\varphi}\right) \\
& -\sum_{i=1}^{2}\left(\frac{\widetilde{g}_{i}\left(p_{i}\right) \dot{\hat{g}}_{i}\left(p_{i}\right)}{\chi_{i}}+\frac{\tilde{\theta}_{i} \dot{\hat{\theta}}_{i}}{\eta_{i}}\right)+\sum_{q_{1}=1}^{N} \Upsilon_{p_{1} q_{1}} V_{1}+\sum_{q_{2}=1}^{N} \Upsilon_{p_{2} q_{2}} V_{2}+\hbar_{2},
\end{aligned}
$$

where $\hbar_{2}=\hbar_{1}+\sum_{i=1}^{2}\left(\left(\delta_{i}^{2} / 2\right)+\left(\bar{\tau}_{i}^{2} / 2\right)\right)$. 
Design the control input $u_{z}$ and $u_{\varphi}$ that are subjects to random actuator faults as

$$
\begin{aligned}
& u_{z}=-\lambda_{1} \gamma_{1}-\frac{\gamma_{2}}{2}-\frac{\gamma_{2} \widehat{\theta}_{1}}{2 \delta_{1}^{2}}-c_{2} \gamma_{2}^{2 a-1} \\
& u_{\varphi}=-\lambda_{3} \gamma_{3}-\frac{\gamma_{4}}{2}-\frac{\gamma_{3} \widehat{\theta}_{2}}{2 \delta_{3}^{2}}-c_{4} \gamma_{4}^{2 a-1}
\end{aligned}
$$

where the parameters $c_{2}$ and $c_{4}$ are positive.

The adaptive law is established as

$$
\dot{\hat{\theta}}_{i}=\frac{\eta_{i}}{2 \delta_{i}^{2}} \gamma_{2 i}^{2}-\kappa_{i} \widehat{\theta}_{i}, \quad i=1,2,
$$

where the designed parameter $\kappa_{i}$ is positive.

Based on (1), (2), and (39), $u_{f}$ and $u_{r}$ are shown as

$$
\begin{aligned}
u_{f} & =\frac{l_{1} u_{z}+u_{\varphi}}{l_{1}+l_{2}}, \\
u_{r} & =\frac{l_{2} u_{z}-u_{\varphi}}{l_{1}+l_{2}}, \\
\Psi_{f}(t) & =\frac{1}{\widehat{g}_{1}\left(l_{1}+l_{2}\right)}\left[l_{1}\left(-\lambda_{1} \gamma_{1}-\frac{\gamma_{2}}{2}-\frac{\gamma_{2} \widehat{\theta}_{1}}{2 \delta_{1}^{2}}-c_{2} \gamma_{2}^{2 a-1}\right)-\lambda_{3} \gamma_{3}-\frac{\gamma_{4}}{2}-\frac{\gamma_{3} \widehat{\theta}_{2}}{2 \delta_{3}^{2}}-c_{4} \gamma_{4}^{2 a-1}\right], \\
\Psi_{r}(t) & =\frac{1}{\widehat{g}_{2}\left(l_{1}+l_{2}\right)}\left[l_{2}\left(-\lambda_{1} \gamma_{1}-\frac{\gamma_{2}}{2}-\frac{\gamma_{2} \widehat{\theta}_{1}}{2 \delta_{1}^{2}}-c_{2} \gamma_{2}^{2 a-1}\right)+\lambda_{3} \gamma_{3}+\frac{\gamma_{4}}{2}+\frac{\gamma_{3} \widehat{\theta}_{2}}{2 \delta_{3}^{2}}+c_{4} \gamma_{4}^{2 a-1}\right] .
\end{aligned}
$$

The updating laws of estimated parameters are established:

$$
\begin{aligned}
& \dot{\hat{g}}_{1}\left(p_{1}\right)=\operatorname{proj}_{\widehat{g}_{1}\left(p_{1}\right)} \underset{\left(\gamma_{2}+l_{1} \gamma_{4}\right) \chi_{1} \psi_{f}(t)-2 c_{g_{1}} \widehat{g}_{1}\left(p_{1}\right)}{ } \begin{cases}0, & \widehat{g}_{1}\left(p_{1}\right)=g_{1 \mathrm{~min}}, \\
\left(\gamma_{2}+l_{1} \gamma_{4}\right) \chi_{1} \psi_{f}(t)-2 c_{g_{1}} \widehat{g}_{1}\left(p_{1}\right), \widehat{g}_{1}\left(p_{1}\right), & \text { others, }\end{cases} \\
& \dot{\hat{g}}_{2}\left(p_{2}\right)=\operatorname{proj}_{\widehat{g}_{2}\left(p_{2}\right)}^{\left(\gamma_{2}-l_{2} \gamma_{4}\right) \chi_{2} \psi_{r}(t)-2 c_{g_{2}} \widehat{g}_{2}\left(p_{2}\right)} \begin{cases}0, & \widehat{g}_{2}\left(p_{2}\right)=g_{2 \mathrm{~min}}, \\
\left(\gamma_{2}-l_{1} \gamma_{4}\right) \chi_{2} \psi_{r}(t)-2 c_{g_{2}} \widehat{g}_{2}\left(p_{2}\right), \widehat{g}_{2}\left(p_{2}\right), & \text { others, }\end{cases}
\end{aligned}
$$

where proj represents the projection operator in order to avoid singular values in the denominator.

Remark 4. The adaptive estimated parameters $\dot{\hat{g}}_{1}\left(p_{1}\right)$ and $\dot{\hat{g}}_{2}\left(p_{2}\right)$ appear in the denominators in (41), which may cause the controllers to fail. Construct the projection form to avoid this situation.

Substituting (39)-(41) into (37), LV can be expressed as

$$
\begin{aligned}
\mathrm{LV} \leq & -\sum_{i=1,3} c_{i}\left[\left(\frac{1-q\left(\gamma_{i}\right)}{2} \log \frac{1}{k_{a i}^{2}-\gamma_{i}^{2}}\right)^{a}+\left(\frac{q\left(\gamma_{i}\right)}{2} \log \frac{1}{k_{b i}^{2}-\gamma_{i}^{2}}\right)^{a}\right] \\
& -\sum_{i=1}^{2} c_{2 i} \gamma_{2 i}^{2 a}+\sum_{i=1}^{2} \frac{c_{g_{i}} \widetilde{g}_{i}\left(p_{i}\right) \widehat{g}_{i}\left(p_{i}\right)}{\chi_{i}}+\frac{\kappa_{i} \widetilde{\theta}_{i} \widehat{\theta}_{i}}{\eta_{i}} \\
& +\sum_{q_{1}=1}^{N} \Upsilon_{p_{1} q_{1}} V_{1}+\sum_{q_{2}=1}^{N} \Upsilon_{p_{2} q_{2}} V_{2}+\hbar_{2} .
\end{aligned}
$$

By Young's inequality $(i=1,2)$, they can obtain

$$
\begin{gathered}
\widetilde{\theta}_{i} \widehat{\theta}_{i} \leq \frac{1}{2} \widetilde{\theta}_{i}^{2}+\frac{1}{2} \theta_{i}^{2}, \\
\widetilde{g}_{i}\left(p_{i}\right) \widehat{g}_{i}\left(p_{i}\right) \leq-\frac{1}{2} \widetilde{g}_{i}^{2}\left(p_{i}\right)+\frac{1}{2} \widehat{g}_{i}^{2}\left(p_{i}\right) .
\end{gathered}
$$

LV can be rewritten as

$$
\begin{aligned}
\mathrm{LV} \leq & -\sum_{i=1,3} c_{i}\left[\left(\frac{1-q\left(\gamma_{i}\right)}{2} \log \frac{1}{k_{a i}^{2}-\gamma_{i}^{2}}\right)^{a}+\left(\frac{q\left(\gamma_{i}\right)}{2} \log \frac{1}{k_{b i}^{2}-\gamma_{i}^{2}}\right)^{a}\right] \\
& -\sum_{i=1}^{2}\left[c_{2 i} \gamma_{2 i}^{2 a}+\frac{c_{g_{i}} \widetilde{g}_{i}^{2}\left(p_{i}\right)}{2 \chi_{i}}+\frac{\kappa_{i} \widetilde{\theta}_{i}^{2}}{2 \eta_{i}}\right] \\
& +\sum_{q_{1}=1}^{N} \Upsilon_{p_{1} q_{1}} V_{1}+\sum_{q_{2}=1}^{N} \Upsilon_{p_{2} q_{2}} V_{2}+\hbar_{3},
\end{aligned}
$$

where $\hbar_{3}=\hbar_{2}+\sum_{i=1}^{2}\left(\left(\kappa_{i} \theta_{i}^{2} / 2 \eta_{i}\right)+\left(c_{g_{i}} g_{i}^{2}\left(p_{i}\right) / 2 \chi_{i}\right)\right)$.

Based on Lemma 3, let $z=\left(\widetilde{\theta}_{i}^{2} / 2 \eta_{i}\right) a=(2 n-1) /(2 n+1)$, $n \in \mathbb{N}, b=1-a, \alpha=1$, and $\mu=a^{(a /(1-a))}$, one has 


$$
\left(\frac{\tilde{\theta}_{i}^{2}}{2 \eta_{i}}\right)^{a} \leq(1-a) \mu+\frac{\tilde{\theta}_{i}^{2}}{2 \eta_{i}} .
$$

Furthermore, we can obtain

$$
-\frac{\widetilde{\theta}_{i}^{2}}{2 \eta_{i}} \leq(1-a) \mu-\left(\frac{\tilde{\theta}_{i}^{2}}{2 \eta_{i}}\right)^{a} .
$$

Applying the inequality (44) and (46) can be expressed as

$$
\begin{aligned}
\mathrm{LV} \leq & -\sum_{i=1,3} c_{i}\left[\left(\frac{1-q\left(\gamma_{i}\right)}{2} \log \frac{1}{k_{a i}^{2}-\gamma_{i}^{2}}\right)^{a}+\left(\frac{q\left(\gamma_{i}\right)}{2} \log \frac{1}{k_{b i}^{2}-\gamma_{i}^{2}}\right)^{a}\right]-\sum_{i=1}^{2}\left[c_{2 i} \gamma_{2 i}^{2 a}+\frac{c_{g_{i}} \widetilde{g}_{i}^{2}\left(p_{i}\right)}{2 \chi_{i}}+\kappa_{i}\left(\frac{\widetilde{\theta}_{i}^{2}}{2 \eta_{i}}\right)^{a}\right] \\
& +\sum_{q_{1}=1}^{N} \Upsilon_{p_{1} q_{1}} V_{1}+\sum_{q_{2}=1}^{N} \Upsilon_{p_{2} q_{2}} V_{2}+\hbar,
\end{aligned}
$$

where $\hbar=\hbar_{3}+(1-a) \mu$.

In addition, the number of passengers and the load conditions of the vehicle are limited. The mass $M$ is bounded which means $\rho_{i}$ is bounded, namely,

$$
\underline{\rho}_{i} \leq \rho_{i} \leq \bar{\rho}_{i}, \quad i=1,2 .
$$

Based on (48),

$$
\begin{aligned}
\mathrm{LV} \leq & -\sum_{i=1,3} c_{i}\left[\left(\frac{1-q\left(\gamma_{i}\right)}{2} \log \frac{1}{k_{a i}^{2}-\gamma_{i}^{2}}\right)^{a}+\left(\frac{q\left(\gamma_{i}\right)}{2} \log \frac{1}{k_{b i}^{2}-\gamma_{i}^{2}}\right)^{a}\right] \\
& -\sum_{i=1}^{2}\left[c_{2 i}\left(2 \underline{\rho}_{i}\right)^{a}\left(\frac{\gamma_{2 i}^{2 a}}{2 \underline{\rho}_{i}}\right)^{a}+\frac{c_{g_{i}} \widetilde{g}_{i}^{2}\left(p_{i}\right)}{2 \chi_{i}}+\kappa_{i}\left(\frac{\widetilde{\theta}_{i}^{2}}{2 \eta_{i}}\right)^{a}\right] \\
& +\sum_{q_{1}=1}^{N} \Upsilon_{p_{1} q_{1}} V_{1}+\sum_{q_{2}=1}^{N} \Upsilon_{p_{2} q_{2}} V_{2}+\hbar .
\end{aligned}
$$

From (16), (49) can be rewritten as

$$
\mathrm{LV} \leq-\ell V^{a}+\hbar+\sum_{q_{1}=1}^{N} \Upsilon_{p_{1} q_{1}} V_{1}+\sum_{q_{2}=1}^{N} \Upsilon_{p_{2} q_{2}} V_{2},
$$

where $\ell=\min \left\{-c_{2}\left(2 \rho_{1}\right)^{a}, c_{4}\left(2 \rho_{2}\right)^{a}, c_{1}, c_{3}, \kappa_{1}, \kappa_{2}, c_{g_{1}}, c_{g_{2}}\right\}$.

The following will apply the discrete expectation to address the random terms.

$$
\lim _{\|x\| \longrightarrow \infty} \inf _{t \geq t_{0}} V=\infty .
$$

There would be a $B$, no matter how big it is; once more than $t>t_{l}$, it would exceed $B$. From (51) in $\left[t_{0}, t_{l}\right],\|x(t)\| \leq B$. (50) is bounded in $\left[t_{0}, t_{l}\right]$.

Then, according to property of discrete expectations, it yields

$$
\begin{aligned}
E(\mathrm{LV}) \leq & \sum_{p_{1}=1}^{N} \sum_{p_{2}=1}^{N} E\left(\mathrm{LV}_{l}\right) \pi_{p_{1}} \pi_{p_{2}} \\
\leq & -\ell \sum_{p_{1}=1}^{N} \sum_{p_{2}=1}^{N} E\left(\mathrm{LV}_{l}^{a}\right) \pi_{p_{1}} \pi_{p_{2}}+\hbar \\
& +\sum_{p_{1}=1}^{N} \sum_{p_{2}=1}^{N} E\left(\sum_{q_{1}=1}^{N} \Upsilon_{p_{1} q_{1}} V_{1 l}+\sum_{q_{2}=1}^{N} \Upsilon_{p_{2} q_{2}} V_{2 l}\right) \pi_{p_{1}} \pi_{p_{2}} \\
\leq & -\ell \sum_{p_{1}=1}^{N} \sum_{p_{2}=1}^{N} E\left(\mathrm{LV}_{l}^{a}\right)+\hbar+N\left(\sum_{k=1}^{2} \max _{p_{k}, q_{k}=1}^{N}\left\{\gamma_{p_{k} q_{k}} \frac{\pi_{p_{k}}}{\pi_{q_{k}}}\right\}\right) E V_{l}^{a} \leq-\left(\ell-\ell_{l}\right) E V_{l}^{a}+\hbar,
\end{aligned}
$$


where $V_{l}=V\left(x\left(t_{l}\right), r_{1}\left(t_{l}\right), r_{2}\left(t_{l}\right)\right), \quad V_{1 l}=\left(x\left(t_{l}\right), p_{2}, q_{1}\right)$, $V_{2 l}=\left(x\left(t_{l}\right), p_{1}, q_{2}\right)$, and $V_{l}^{a}=V^{a}\left(x\left(t_{l}\right), r_{1}\left(t_{l}\right), r_{2}\left(t_{l}\right)\right)$. The stability distribution law of Markovian variable $r_{i}\left(t_{l}\right)$ is $\pi_{p_{i}}=\left(\pi_{1}, \pi_{2}, \ldots, \pi_{N}\right) . \quad \sum_{j=1}^{N} \pi_{j}=1, \quad$ and $\quad \pi_{j}>0$. $\ell_{l}=N\left(\sum_{k=1}^{2} \max _{p_{k}, q_{k}=1}^{N}\left\{\gamma_{p_{k} q_{k}}\left(\pi_{p_{k}} / \pi_{q_{k}}\right)\right\}\right)$. Various parameters associated with the selection need to be satisfied, $\left(\ell-\ell_{l}\right)>0$.

$$
\begin{aligned}
E V_{l}^{a} & =V_{t_{0}}^{a}+E \int_{t_{0}}^{t_{l}} e^{\left(\ell-\ell_{l}\right) s} \mathrm{LV}_{s}^{a} \mathrm{~d} s \\
& \leq V_{t_{0}}^{a}+\hbar\left(t_{l}-t_{0}\right)-\left(\ell-\ell_{l}\right) \int_{t_{0}}^{t_{l}} E V_{s}^{a} \mathrm{~d} s \\
& \leq V_{t_{0}}^{a}+\hbar\left(t_{l}-t_{0}\right),
\end{aligned}
$$

where $V_{t_{0}}^{a}=V^{a}\left(x\left(t_{0}\right), r_{1}\left(t_{0}\right), r_{2}\left(t_{0}\right)\right)$.

For $\forall x\left(t_{0}\right)$ and $r_{1}\left(t_{0}\right), r_{2}\left(t_{0}\right) \in S$, there is a unique solution $x(t)$ in the closed-loop systems.

$$
\begin{aligned}
& E\left(e^{\left(\ell-\ell_{l}\right) t_{l}} V_{l}^{a}\right)-E\left(e^{\left(\ell-\ell_{l}\right) t_{0}} V_{t_{0}}^{a}\right) \\
& =E\left(\int_{t_{0}}^{t_{l}} e^{\left(\ell-\ell_{l}\right) s} \mathrm{~L} V_{s}^{a} \mathrm{~d} s\right)+\left(\ell-\ell_{l}\right) E\left(\int_{t_{0}}^{t_{l}} e^{\left(\ell-\ell_{l}\right) s} \mathrm{LV}_{s}^{a} \mathrm{~d} s\right) \\
& \leq-\left(\ell-\ell_{l}\right) E\left(\int_{t_{0}}^{t_{l}} e^{\left(\ell-\ell_{l}\right) s} \mathrm{~L} V_{s}^{a} \mathrm{~d} s\right)+\int_{t_{0}}^{t_{l}} \hbar e^{\left(\ell-\ell_{l}\right) s} \mathrm{~d} s \\
& \quad+\left(\ell-\ell_{l}\right) E\left(\int_{t_{0}}^{t_{l}} e^{\left(\ell-\ell_{l}\right) s} V_{s}^{a} \mathrm{~d} s\right) \\
& E\left(e^{\left(\ell-\ell_{l}\right) \ell_{l}} V_{l}^{a}\right) \leq e^{\left(\ell-\ell_{l}\right) t_{0}} V_{t_{0}}^{a}+\frac{\hbar}{\ell-\ell_{l}}\left(e^{\left(\ell-\ell_{l}\right) t}-e^{\left(\ell-\ell_{l}\right) t_{0}}\right) \\
& E\left(V_{l}^{a}\right) \leq e^{-\left(\ell-\ell_{l}\right)\left(t-t_{0}\right)} V_{t_{0}}^{a}+\frac{\hbar}{\ell-\ell_{l}}\left(1-e^{-\left(\ell-\ell_{l}\right)\left(t-t_{0}\right)}\right) \\
& \leq V_{t_{0}}^{a}+\frac{\hbar}{\ell-\ell_{l}} .
\end{aligned}
$$

From the Lemma 2, it can be concluded that all the signals can be guaranteed SGPFS. Meanwhile, the constraints of two different output factors are bounded.

Theorem 1. The finite-time FTC control can be implemented by designing the virtual signal (17), the controller in (40), and the updating law (39) and (41). Meantime, by selecting appropriate design parameters, the tracking error of the systems can be arbitrarily small in a finite-time.

Proof. Through Lemma 2 in [37] and inequality (54), we can acquire that the trajectories of the all signals that satisfy $V^{a}(\gamma(t), \tilde{\theta}(t)) \leq\left(\hbar /\left(1-\theta_{0}\right) \ell_{12}\right)$, for any $t \geq T_{\text {reach }}$, in finite time, where $T_{\text {reach }}$ is defined as $T_{\text {reach }}=(1 /(1-$ $\left.\left.\theta_{0}\right) \ell_{12}\right)\left(V^{1-a}\left(\gamma(0), \theta_{1}(0)\right)\right)-\left(\hbar /\left(1-\theta_{0}\right) \ell_{12}\right)^{(1-a) / a} \quad$ with $\gamma(0)=\left[\gamma_{1}(0), \gamma_{2}(0)\right]^{T}$. In other words, all signals of the studied closed system are SGPFS.

Furthermore, the following result can be obtained, $i=1,3 . \quad\left|x_{i}-y_{i d}\right| \leq k_{i}\left[1-e^{-2\left(\hbar /\left(1-\theta_{0}\right) \ell_{12}\right)^{1 / a}}\right]^{(1 / 2)}$, where $k_{i}=\min \left\{k_{a i}, k_{b i}\right\}$, In addition, the tracking error $\gamma_{i}$ remains in a small neighborhood of the origin by finite-time control.

When $q\left(\gamma_{i}\right)=1$ and $\gamma_{i} \geq 0$, we can get $\gamma_{i} \leq k_{b i}\left[1-e^{-2\left(\hbar /\left(1-\theta_{0}\right) \ell_{12}\right)^{1 / a}}\right]^{(1 / 2)} \leq k_{b i}$. Thus, when $q\left(\gamma_{i}\right)=0$ and $\gamma_{i}<0$, it can obtain $\gamma_{i} \geq-k_{a i}[1-$ $\left.e^{-2\left(\hbar /\left(1-\theta_{0}\right) \ell_{12}\right)^{1 / a}}\right]^{(1 / 2)} \geq-k_{a i}$. By using $-\underline{B}_{i 1} \leq y_{i d} \leq \bar{B}_{i 1}$, it yields $-\underline{k}_{c i} \leq-k_{a i}+y_{i d} \leq x_{i} \leq k_{b i}+y_{i d} \leq \bar{k}_{c i}$. In short, $x_{i}$ is constrained in its limit.

3.2. Zero Dynamics and Performance Analysis. Through the analysis in Section 3.1, the signals $x_{i}$ and $\widehat{\theta}_{j}(i=1,2,3,4, j=1,2)$ are bounded. Then, we are going to prove the zero dynamics that consist of the other four states $x_{5}, x_{6}, x_{7}$, and $x_{8}$.

Therefore, substituting (38)-(41) into (5), let $\gamma_{1}=\gamma_{2}=\gamma_{3}=\gamma_{4}=0$. It yields the following form:

$$
\dot{Z}=A Z+B_{1} D_{1}+B_{0} D_{0}+W,
$$

where

$$
\begin{aligned}
Z & =\left[x_{5}, x_{6}, x_{7}, x_{8}\right]^{T}, \\
D_{0} & =\left[D_{01}, \dot{D}_{01}, D_{02}, \dot{D}_{02}\right]^{T}, \\
D_{1} & =\left[D_{f}-D_{1}, \dot{D}_{f}-\dot{D}_{1}, D_{r}-D_{2}, \dot{D}_{r}-\dot{D}_{2}\right]^{T},
\end{aligned}
$$$$
A=\left[\begin{array}{cccc}
0 & 1 & 0 & 0 \\
\frac{-k_{f 2}}{m_{f}} & \frac{-b_{f 2}}{m_{f}} & 0 & 0 \\
0 & 0 & 1 & 0 \\
0 & 0 & \frac{-k_{r 2}}{m_{r}} & \frac{-b_{r 2}}{m_{r}}
\end{array}\right],
$$$$
B_{0}=\left[\begin{array}{cccc}
0 & 0 & 0 & 0 \\
\frac{k_{f 2}}{m_{f}} & \frac{b_{f 2}}{m_{f}} & 0 & 0 \\
0 & 0 & 0 & 0 \\
0 & 0 & \frac{k_{r 2}}{m_{r}} & \frac{k_{r 2}}{m_{r}}
\end{array}\right],
$$ 


$$
\begin{aligned}
B_{1} & =\left[\begin{array}{cccc}
0 & 0 & 0 & 0 \\
\frac{\tilde{g}_{1} k_{f 2}}{m_{f} \widehat{g}_{1}} & \frac{\widetilde{g}_{1} b_{f 2}}{m_{f} \widehat{g}_{1}} & 0 & 0 \\
0 & 0 & 0 & 0 \\
0 & 0 & \frac{\tilde{g}_{2} k_{r 2}}{m_{r} \widehat{g}_{2}} & \frac{\tilde{g}_{2} b_{r 2}}{m_{r} \widehat{g}_{2}}
\end{array}\right], \\
w & =\left[\begin{array}{c}
0 \\
\frac{-l_{2} w_{1}-w_{2}}{m_{f}\left(l_{1}+l_{2}\right)} \\
0 \\
w_{1}= \\
\frac{-l_{2} w_{1}+w_{2}}{m_{r}\left(l_{1}+l_{2}\right)}
\end{array}\right], \\
w_{2} & =-\lambda_{3} \gamma_{1}-\frac{1}{2} \gamma_{2}-\frac{1}{2} \gamma_{4}-\frac{\gamma_{2}}{2 \delta_{1}^{2}} \widehat{\theta}_{1}-c_{2} \gamma_{2}^{2 l-1} \\
2 \delta_{3}^{2} & \hat{\theta}_{2}-c_{4} \gamma_{4}^{2 l-1} .
\end{aligned}
$$

Notable is, due to the boundedness of $x_{i}$ and $\widehat{\theta}_{j}(i=1,2,3,4, j=1,2), w_{1}$ and $w_{2}$ are bounded, namely, $\left\|w_{i}\right\| \leq \omega_{i}$ for the positive parameter $\omega_{i}(i=1,2)$. Furthermore, it means that $W$ satisfies $\|W\| \leq \bar{W}$, for the parameter $\bar{W}>0$. Meanwhile, according to Assumptions 1 and 2, D satisfies $\|D\| \leq \bar{D}$, for the parameter $\bar{D}>0$. Choosing the positive matrixes $P$ and $Q$ can ensure the following equality holds, namely,

$$
A^{T} P+P^{T} A=-Q
$$

Define

$$
V_{Z}=Z^{T} P Z
$$

where $V_{Z}=V_{Z}\left(Z, p_{1}, p_{2}\right)$, and $P$ is a positive matrix.

According to the infinitesimal generator in [50], (58) can be calculated at the mode of $\left(r_{1}(t), r_{2}(t)\right)=\left(p_{1}, p_{2}\right)$ as

$$
\mathrm{LV}_{Z}=\dot{Z}^{T} P Z+Z^{T} P \dot{Z}+\sum_{q_{1}=1}^{N} \Upsilon_{p_{1} q_{1}} Z_{1}+\sum_{q_{2}=1}^{N} \Upsilon_{p_{2} q_{2}} Z_{2}
$$

where $Z_{1}=Z_{1}\left(Z, p_{2}, q_{1}\right)$, and $Z_{2}=Z_{2}\left(Z, p_{1}, q_{2}\right)$.

$$
\begin{aligned}
& \mathrm{LV}_{Z}=\left(A Z+B_{1} D_{1}+B_{0} D_{0}+W\right)^{T} P Z \\
& Z^{T} P\left(A Z+B_{1} D_{1}+B_{0} D_{0}+W\right)+\sum_{q_{1}=1}^{N} \Upsilon_{p_{1} q_{1}} Z_{1}+\sum_{q_{2}=1}^{N} \Upsilon_{p_{2} q_{2}} Z_{2} \\
&= Z^{T}\left(A^{T} P+P^{T} A\right) Z+2 Z^{T} P B_{1} D_{1}+2 Z^{T} P B_{0} D_{0}+2 Z^{T} P W \\
&+\sum_{q_{1}=1}^{N} \Upsilon_{p_{1} q_{1}} Z_{1}+\sum_{q_{2}=1}^{N} \Upsilon_{p_{2} q_{2}} Z_{2} .
\end{aligned}
$$

Based on Young's inequality, it obtains

$$
\begin{gathered}
2 Z^{T} P B_{0} D_{0} \leq \frac{\lambda_{\max }\left(P B_{0}^{T} B_{0} P\right)}{\vartheta_{0}}\|Z\|^{2}+\vartheta_{0} \bar{D}_{0}^{2}, \\
2 Z^{T} P B_{1} D_{1} \leq \frac{\lambda_{\max }\left(P B_{1}^{T} B_{1} P\right)}{\vartheta_{1}}\|Z\|^{2}+\vartheta_{1} \bar{D}_{1}^{2}, \\
2 Z^{T} P W \leq \frac{\lambda_{\max }(P P)}{\vartheta_{2}}\|Z\|^{2}+\vartheta_{2} \bar{W}^{2},
\end{gathered}
$$

where the parameters $\vartheta_{0}, \vartheta_{1}$, and $\vartheta_{2}$ are positive.

Combining (57) and (61), (60) can be rewritten as

$$
\begin{aligned}
\mathrm{LV}_{Z}= & -Z^{T} \mathrm{Q} Z+\frac{\lambda_{\max }\left(P B_{0}^{T} B_{0} P\right)}{\vartheta_{0}}\|Z\|^{2}+\frac{\lambda_{\max }\left(P B_{1}^{T} B_{1} P\right)}{\vartheta_{1}}\|Z\|^{2} \\
& +\frac{\lambda_{\max }(P P)}{\vartheta_{2}}\|Z\|^{2}+\hbar_{Z}+\sum_{q_{1}=1}^{N} \Upsilon_{p_{1} q_{1}} Z_{1}+\sum_{q_{2}=1}^{N} \Upsilon_{p_{2} q_{2}} Z_{2} \\
\leq & -Z^{T}\left(Q-\frac{\lambda_{\max }\left(P B_{0}^{T} B_{0} P\right)}{\vartheta_{0}}-\frac{\lambda_{\max }\left(P B_{1}^{T} B_{1} P\right)}{\vartheta_{1}}-\frac{\lambda_{\max }(P P)}{\vartheta_{2}}\right) Z+\hbar_{Z}+\sum_{q_{1}=1}^{N} \Upsilon_{p_{1} q_{1}} Z_{1}+\sum_{q_{2}=1}^{N} \Upsilon_{p_{2} q_{2}} Z_{2} \\
L V_{Z} \leq & -\ell_{Z} V_{Z}+\hbar_{Z}+\sum_{q_{1}=1}^{N} \Upsilon_{p_{1} q_{1}} Z_{1}+\sum_{q_{2}=1}^{N} \Upsilon_{p_{2} q_{2}} Z_{2},
\end{aligned}
$$

where 


$$
\begin{aligned}
& \hbar_{Z}=\vartheta_{0} \bar{D}_{0}^{2}+\vartheta_{1} \bar{D}_{1}^{2}+\vartheta_{2} \bar{W}^{2}, \\
& \ell_{Z}=\lambda_{\min } P^{-(1 / 2)} Q P^{-(1 / 2)}-\frac{\lambda_{\max }(P)}{\vartheta_{2}}-\frac{\lambda_{\max }\left(P^{(1 / 2)} B_{0}^{T} B_{0} P^{(1 / 2)}\right)}{\vartheta_{0}}-\frac{\lambda_{\max }\left(P^{(1 / 2)} B_{1}^{T} B_{1} P^{(1 / 2)}\right)}{\vartheta_{1}} .
\end{aligned}
$$

Then, same as the above discrete expectations, (62) yields

$$
\mathrm{EV}_{Z} \leq e^{-\ell_{0}\left(t-t_{0}\right)} V_{Z_{0}}+\frac{\hbar_{Z}}{\ell_{0}}\left(1-e^{-\ell_{0}\left(t-t_{0}\right)}\right),
$$

where $\ell_{0}=\ell_{Z}-\ell_{l}$, and $V_{Z_{0}}\left(Z\left(t_{0}\right), r_{1}\left(t_{0}\right), r_{2}\left(t_{0}\right)\right)$.
According to Lemma 2, all the signals of the zero dynamics are bounded.

Now, the control objectives will be proved. The dynamic tire loads are given as

$$
\begin{aligned}
& \left|F_{\mathrm{tf}}+F_{\mathrm{bf}}\right| \leq\left(k_{f_{2}}+b_{f_{2}}\right)\left(\frac{\left(V_{Z_{0}}+\hbar_{3} / \ell_{Z}\right)}{\lambda_{\min }(P)}\right)^{(1 / 2)}+k_{f_{2}} \bar{D}_{11}+b_{f_{2}} \bar{D}_{12}, \\
& \left|F_{\mathrm{tr}}+F_{\mathrm{br}}\right| \leq\left(k_{r_{2}}+b_{r_{2}}\right)\left(\frac{\left(V_{Z_{0}}+\hbar_{3} / \ell_{Z}\right)}{\lambda_{\min }(P)}\right)^{(1 / 2)}+k_{r_{2}} \bar{D}_{21}+b_{r_{2}} \bar{D}_{22} .
\end{aligned}
$$

Thus, selecting appropriate parameters $\vartheta_{1}$ and $\vartheta_{2}$ can ensure $\left(k_{f_{2}}+b_{f_{2}}\right) \vartheta_{3}+k_{f_{2}} \bar{D}_{11}+b_{f_{2}} \bar{D}_{12} \leq F_{f}, \quad\left(k_{r_{2}}+b_{r_{2}}\right) \vartheta_{3}+$ $k_{r_{2}} \bar{D}_{21}+b_{r_{2}} \bar{D}_{22} \leq F_{r}$ hold, that is, (6) holds.
Finally, we are going to learn about the suspension deflection performances. According to the analysis in Section 3.1 and 3.2, one has

$$
\begin{aligned}
& \left|\Delta \bar{D}_{f}\right| \leq x_{1}+l_{2} \sin x_{3}+x_{5} \leq x_{1}+l_{2} x_{3}+x_{5} \leq k_{c_{1}}+l_{2} k_{c_{2}}+\left(\frac{\left(V_{Z_{0}}+\hbar_{3} / \ell_{Z}\right)}{\lambda_{\min }(P)}\right)^{(1 / 2)}, \\
& \left|\Delta \bar{D}_{r}\right| \leq x_{1}+l_{2} \sin x_{3}+x_{7} \leq x_{1}+l_{2} x_{3}+x_{7} \leq k_{c_{1}}+l_{2} k_{c_{2}}+\left(\frac{\left(V_{Z_{0}}+\hbar_{3} / \ell_{Z}\right)}{\lambda_{\min }(P)}\right)^{(1 / 2)} .
\end{aligned}
$$

Thus, let $k_{c_{1}}+l_{2} k_{c_{2}}+\left(\left(V_{Z_{0}}+\hbar_{3} / \ell_{Z}\right) / \lambda_{\min }(P)\right)^{(1 / 2)} \leq$ $\Delta \bar{D}_{f}$ and $k_{c_{1}}+l_{2} k_{c_{2}}+\left(\left(V_{Z_{0}}+\hbar_{3} / \ell_{Z}\right) / \lambda_{\text {min }}(P)\right)^{(1 / 2)} \leq \Delta \bar{D}_{r}$, and we have $\left|\Delta \bar{D}_{f}\right| \leq \Delta \bar{D}_{f}$ and $\left|\Delta \bar{D}_{r}\right| \leq \Delta \bar{D}_{r}$, which imply the suspension deflection performances can be achieved.

Remark 5. The works $[38,39]$ mainly focused on the finitetime control problem of quarter active suspension systems. Compared with the foregoing, this study not only addresses the finite-time control of half-vehicle but also further studies constraints of displacement and pitch angle of the car body. Particularly, the random faults between different actuators are studied.

\section{Simulation Example}

In this section, an example is given to demonstrate the established approach for half-vehicle ASSs. In order to facilitate the implement of the simulation, the nonlinear stiffness and dampers are given in [6]

$$
\begin{aligned}
& F_{\mathrm{sf}}=k_{f 01} \Delta D_{f}+k_{f 02} \Delta D_{f}^{3}, \\
& F_{\mathrm{sr}}=k_{r 01} \Delta D_{r}+k_{r 02} \Delta D_{r}^{3}, \\
& F_{\mathrm{tf}}=k_{f 2}\left(D_{1}-D_{01}\right), \\
& F_{\mathrm{tr}}=k_{r 2}\left(D_{2}-D_{02}\right), \\
& F_{\mathrm{bf}}=b_{f 2}\left(\dot{D}_{1}-\dot{D}_{01}\right), \\
& F_{\mathrm{br}}=b_{r 2}\left(\dot{D}_{2}-\dot{D}_{02}\right), \\
& F_{\mathrm{df}}=\left\{\begin{array}{l}
d_{e 1} \Delta \dot{z}_{f}, \\
d_{c 1} \Delta \dot{z}_{f},
\end{array} F_{\mathrm{dr}}=\left\{\begin{array}{l}
d_{e 2} \Delta \dot{z}_{r}, \\
d_{c 2} \Delta \dot{z}_{r},
\end{array}\right.\right.
\end{aligned}
$$

where $k_{f 01}$ and $k_{f 02}$ are the stiffness coefficients of linear and nonlinear terms for the front active suspension systems; $k_{r 01}$ and $k_{r 02}$ are the stiffness coefficients of linear and nonlinear terms for the rear active suspension systems; $k_{f 2}$ and $b_{f 2}$ stand for the damping coefficients of front and rear wheels; $k_{r 2}$ and $b_{r 2}$ denote the damping coefficients of front and rear wheels; $d_{e 1}$ and $d_{c 1}$ are the segmented coefficients of front damper of active suspension systems; and $d_{e 2}$ and $d_{c 2}$ are the 


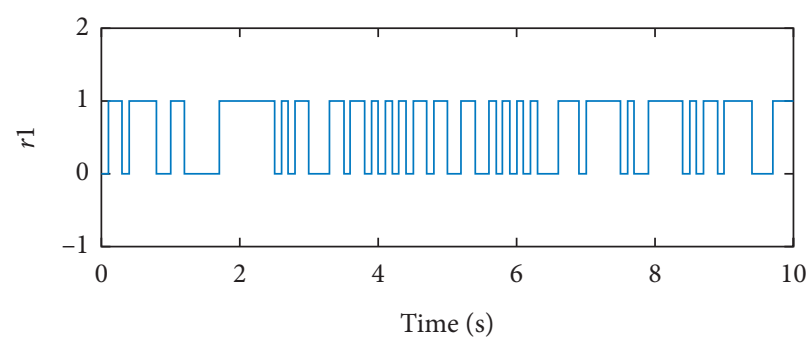

(a)

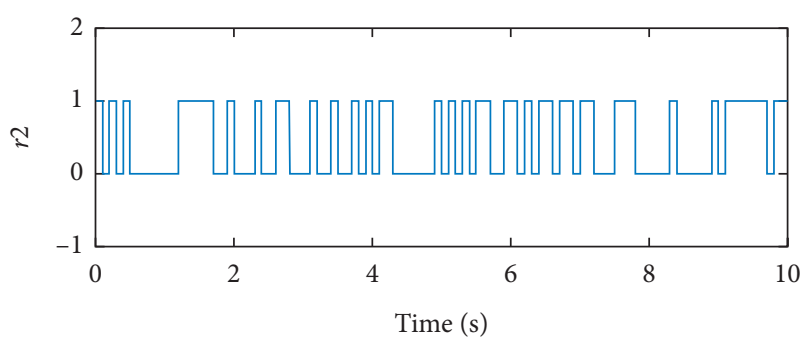

(b)

Figure 2: The curves of $r_{1}(t)$ and $r_{2}(t)$.

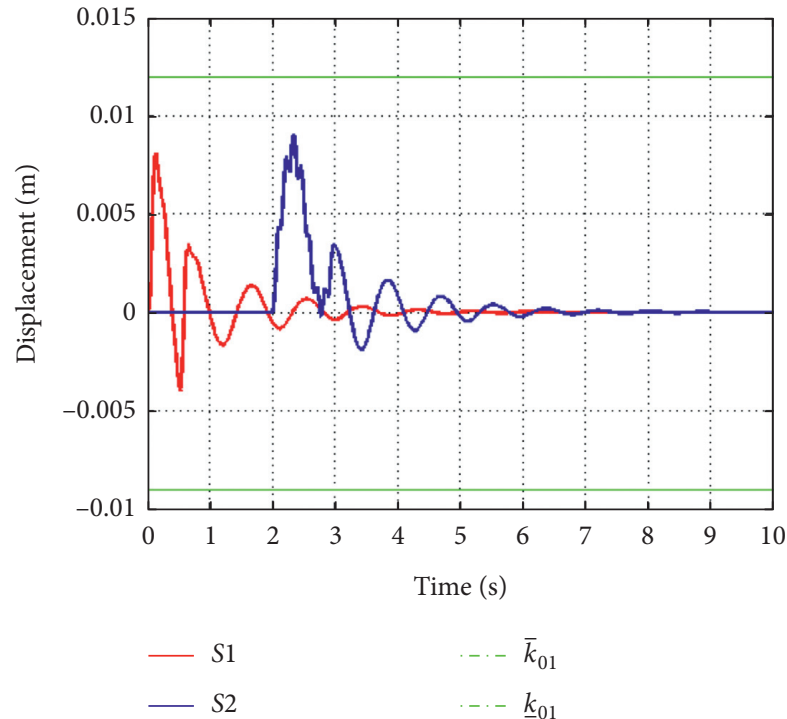

Figure 3: The vertical motion displacement $x_{1}$.

segmented coefficients of front damper of active suspension systems.

The road profile is an important aspect of affecting suspension performance. Thus, two different road inputs are carried out and given as follows:

S1: the road input of the front wheel is described as

$$
D_{01}= \begin{cases}0.5 h\left[1+\sin \left(\frac{2 \pi v}{L} t\right)\right], & t_{1} \leq t \leq t_{h}, \\ 0, & \text { otherwise, }\end{cases}
$$

where $h$ and $L$ are the height and the length of the bump, and $v$ is the velocity of the vehicle. The road input $D_{02}$ for the rear wheel is implemented as $D_{02}(t)=D_{01}(t-\tau)$. Suppose $h=0.05 \mathrm{~m}, L=2.5 \mathrm{~m}$, $v=18 \mathrm{~km} / \mathrm{h}, t_{1}=2, t_{h}=2.5$, and time delay $\tau=0.5$.

$S 2$ : the road input of the front wheel is presented as

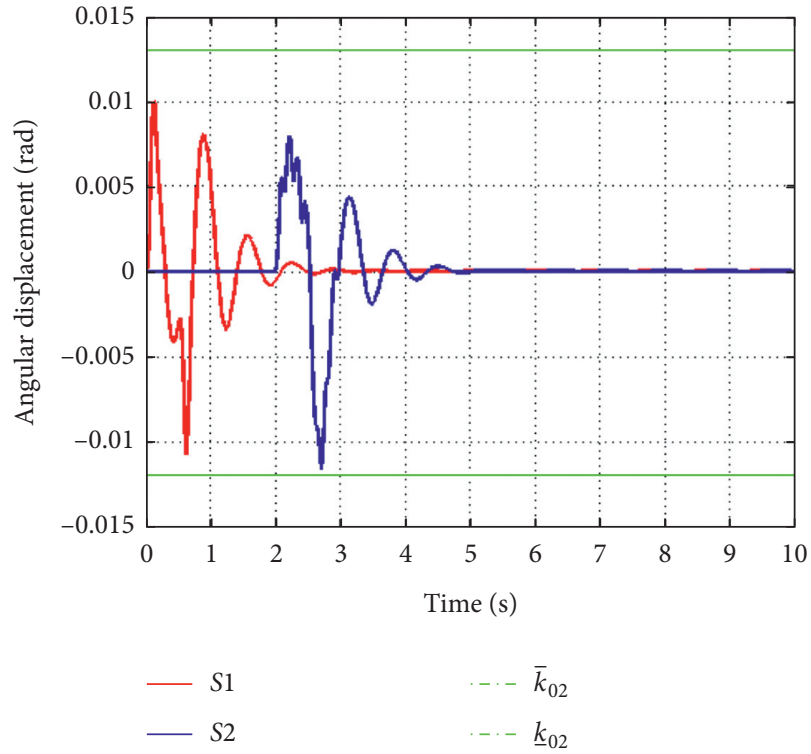

Figure 4: The pitch angle $\varphi$.

$$
D_{01}= \begin{cases}0.5 h_{b}\left[1+\sin \left(\frac{2 \pi v_{b}}{L} t\right)\right], & t_{1} \leq t \leq \frac{L}{v_{b}}, \\ 0, & t>\frac{L}{v_{b}},\end{cases}
$$

where $h_{b}=0.08 \mathrm{~m}, L=2.5 \mathrm{~m}$, and $v_{b}=25 \mathrm{~km} / \mathrm{h}$. The road input $D_{02}$ is carried out the same as $S 1$.

The parameters in half-vehicle active suspension systems are briefly illustrated: $M=1200 \mathrm{~kg}, \quad I=1000 \mathrm{kgm}^{2}$, $m_{f}=m_{r}=100 \mathrm{~kg}, \quad k_{f_{01}}=k_{r_{01}}=15000 \mathrm{~N} / \mathrm{m}$, $k_{f_{02}}=k_{r_{02}}=1000 \mathrm{~N} / \mathrm{m}, \quad k_{f_{2}}=200000 \mathrm{~N} / \mathrm{m}, \quad l_{1}=1.5 \mathrm{~m}$, $k_{r_{2}}=150000 \mathrm{~N} / \mathrm{m}, \quad b_{f_{2}}=1500 \mathrm{Ns} / \mathrm{m}, \quad b_{r_{2}}=2000 \mathrm{Ns} / \mathrm{m}$, $d_{e 1}^{2}=d_{e 2}=1500 \mathrm{Ns} / \mathrm{m}, \quad d_{c 1}=d_{c 2}=1200 \mathrm{Ns} / \mathrm{m}, \quad$ and $l_{2}=1.2 \mathrm{~m}$. The control parameters in this work are given as follows: $\eta_{1}=4, \eta_{2}=6, \delta_{1}=3, \delta_{2}=2, \kappa_{1}=30, \kappa_{2}=40$, $c_{1}=c_{3}=50, \quad c_{2}=c_{4}=100, \quad a=299 / 300, \quad k_{a_{1}}=0.006$, 


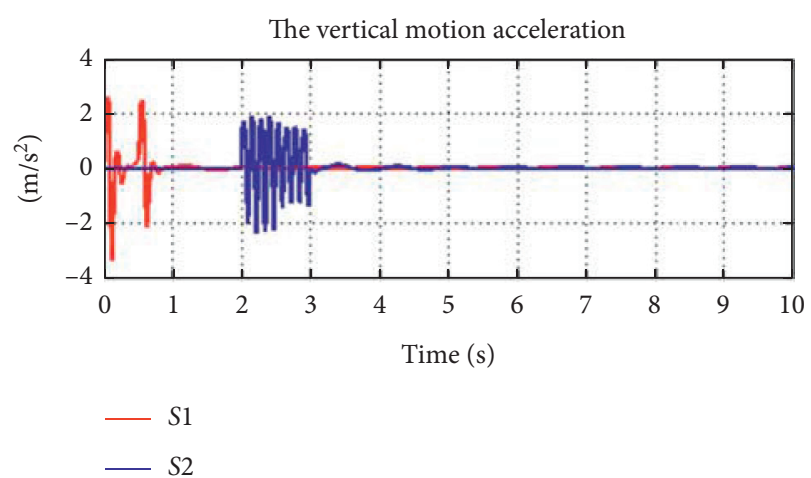

(a)

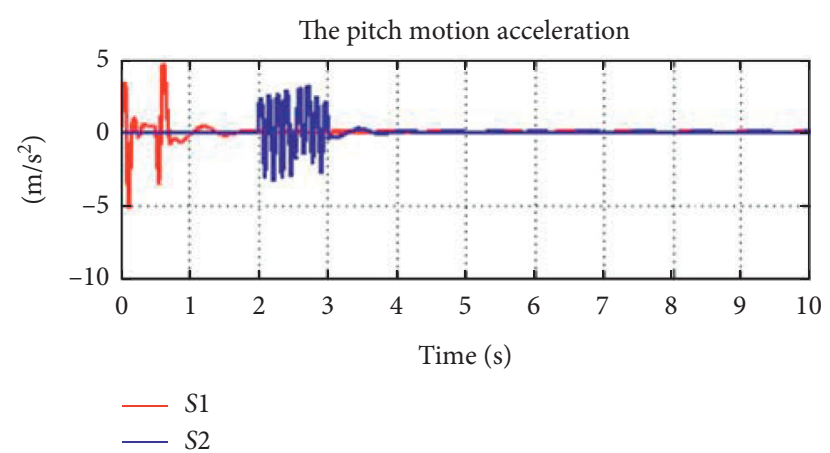

(b)

FIgURE 5: The accelerations of active suspension systems motion.

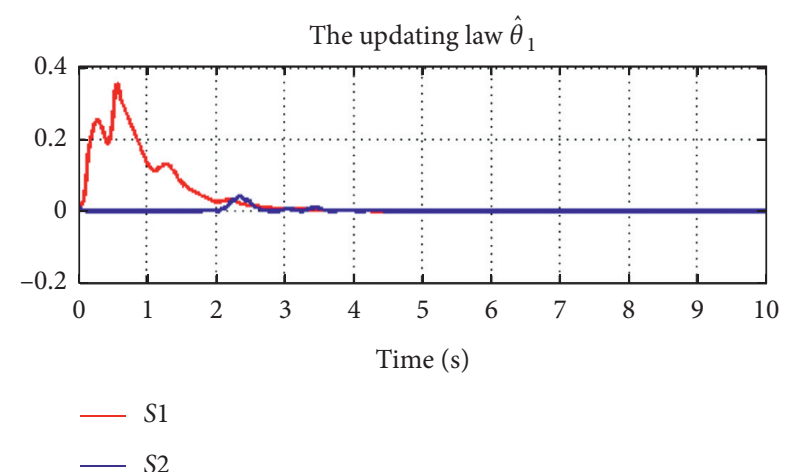

(a)

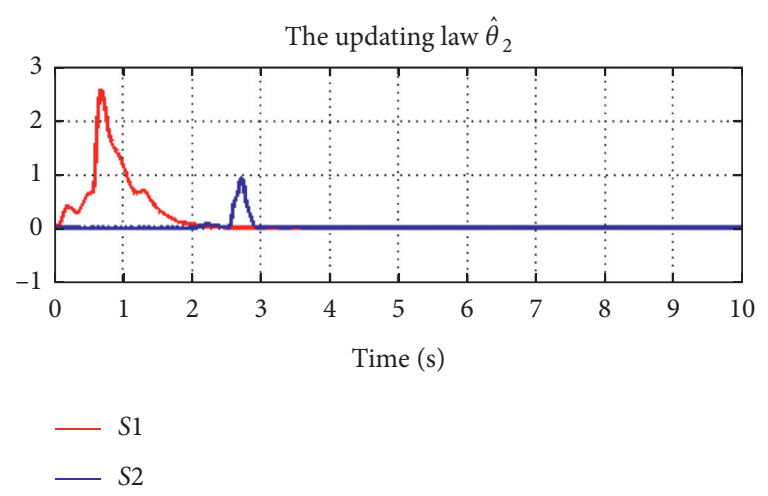

(b)

FIgUre 6: Trajectories of $\widehat{\theta}_{i}(i=1,2)$.

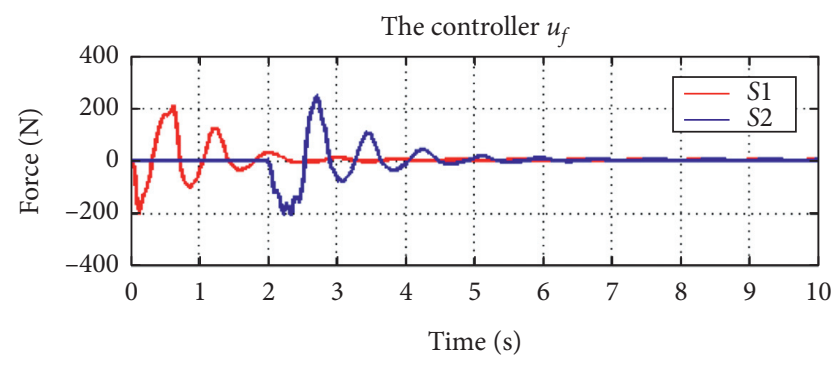

(a)

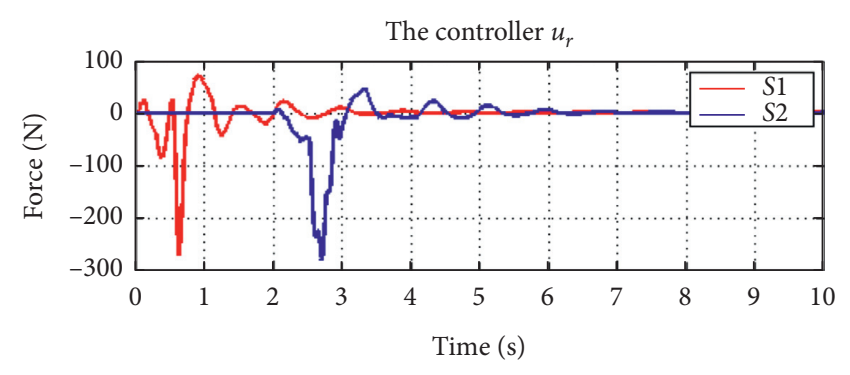

(b)

FIgURE 7: The controllers with fault tolerant.

$k_{b_{1}}=0.01, k_{a_{2}}=0.012, k_{b_{2}}=0.011, \bar{k}_{c_{1}}=0.012, \underline{k}_{c_{1}}=0.009$, $\bar{k}_{c_{2}}=0.013, \underline{k}_{c_{2}}=0.012$, and the rest ones are assumed as zeros. In simulation examples, the initial state values are given as $x(0)=[0,0,0,0,0,0,0,0]^{T}, S\{0,1\}$. For $r_{1}(t)$ : $\left(\gamma_{p q}\right)_{2 \times 2}=\left[\begin{array}{cc}-4 & 4 \\ 3 & -3\end{array}\right] ; r_{2}(t):\left(\gamma_{p q}\right)_{2 \times 2}=\left[\begin{array}{cc}-3 & 3 \\ 4 & -4\end{array}\right]$.

For the cases $S 1$ and $S 2$, the curves of $r_{1}(t)$ and $r_{2}(t)$ are shown in Figure 2; the trajectories of $x_{1}$ which illustrates the displacement of vertical motion are shown in Figure 3. It can be seen that the displacement $x_{1}$ does not exceeds its constraint bounded.
The pitch angle $\varphi$ is constrained under its bounded and can stabilize quickly in a finite time, as shown in Figure 4. Figure 5 shows the accelerations of vertical motion and pitch motion. It is observed that the maximums of vertical motion and pitch motion are $4 \mathrm{~m} / \mathrm{s}^{2}$ and $5 \mathrm{~m} / \mathrm{s}^{2}$, respectively. In addition, the stabilization of these motions is achieved in $1 \mathrm{sec}$ for cases $S 1$ and $S 2$. The purpose of Figure 6 is to show the trajectories of $\widehat{\theta}_{1}$ and $\widehat{\theta}_{2}$. It indicates that for cases $S 1$ and $S 2$, they can converge to zero in a very short period of time. Figure 7 shows the controllers $u_{f}$ and $u_{r}$, and we can observe that the control forces are calculated about $300 \mathrm{~N}$. It means that the designed controllers play a key role in controlling 


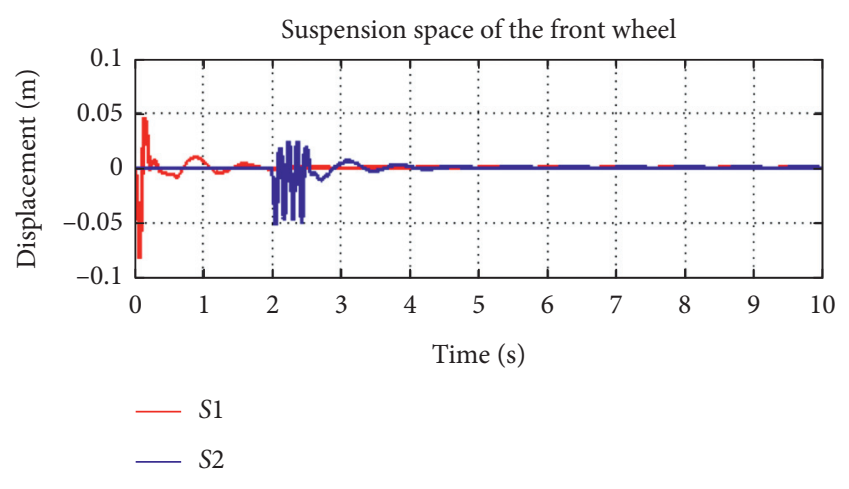

(a)

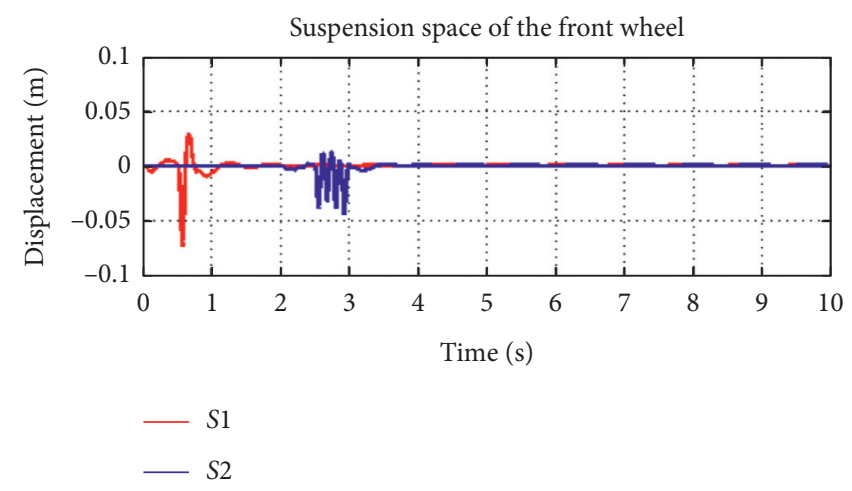

(b)

Figure 8: The suspension spaces.

the variation of active suspension systems. Figure 8 shows that the front wheel and rear wheel of the suspension spaces are all within the bounds. Through the above analysis, the proposed control schemes could be used to help reduce the variation of active suspension systems and achieve a good performance in a finite time.

\section{Conclusions}

In order to achieve good suspension performances, the finite-time control for half active suspension systems have been discussed in this study. The asymmetric BLFs have been employed to constrain vehicle body's displacement and pitch angle in a safety range. Then, utilizing the finite-time control design theory, the adaptive controllers were proposed for vertical motion and pitch motion, respectively. Finally, the effectiveness of designed schemes is demonstrated with the help of results of simulation examples. On the one hand, in the aspect of automobile research, we would further study the battery problem combined with the distributed controller of the multiagent consistency algorithm and apply it to distributed generators in the Energy Internet such as $[56,57]$. On the other hand, we apply this method in the pipeline network system such as [58] and optimal deployment of agents in the pipeline network system in [59]. The study of control problem in the pipeline network system will be an interesting research topic.

\section{Data Availability}

The data used to support the findings of this study are included within the article.

\section{Conflicts of Interest}

The authors declare that there are no conflicts of interest.

\section{Acknowledgments}

This work was supported in part by the National Natural Science Foundation of China (61473139 and 61903168), Provincial Key Research and Development Projects (JH2/
10200002), and Provincial Scientific Research Fund of Education Department (JJL202015407).

\section{References}

[1] H. Pan, W. Sun, X. Jing, H. Gao, and J. Yao, "Adaptive tracking control for active suspension systems with non-ideal actuators," Journal of Sound and Vibration, vol. 399, pp. 2-20, 2017.

[2] W. Sun, H. Gao, and O. Kaynak, "Vibration isolation for active suspensions with performance constraints and actuator saturation," IEEE/ASME Transactions on Mechatronics, vol. 20, no. 2, pp. 675-683, 2015.

[3] S. Kilicaslan, "Control of active suspension system considering nonlinear actuator dynamics," Nonlinear Dynamics, vol. 91, no. 2, pp. 1383-1394, 2018.

[4] W. Sun, H. Gao, and O. Kaynak, "Adaptive backstepping control for active suspension systems with hard constraints," IEEE/ASME Transactions on Mechatronics, vol. 18, no. 3, pp. 1072-1079, 2012.

[5] V. S. Deshpande, P. D. Shendge, and S. B. Phadke, "Nonlinear control for dual objective active suspension systems," IEEE Transactions on Intelligent Transportation Systems, vol. 18, no. 3, pp. 656-665, 2016.

[6] F. Zhao, S. S. Ge, F. Tu, Y. Qin, and M. Dong, "Adaptive neural network control for active suspension system with actuator saturation," IET Control Theory \& Applications, vol. 10, no. 14, pp. 1696-1705, 2016.

[7] G. Wang, C. Chen, and S. Yu, "Robust non-fragile finitefrequency Ho static output-feedback control for active suspension systems," Mechanical Systems and Signal Processing, vol. 91, pp. 41-56, 2017.

[8] S.-A. Chen, J.-C. Wang, M. Yao, and Y.-B. Kim, "Improved optimal sliding mode control for a non-linear vehicle active suspension system," Journal of Sound and Vibration, vol. 395, pp. 1-25, 2017.

[9] C. Hua, J. Chen, Y. Li, and L. Li, "Adaptive prescribed performance control of half-car active suspension system with unknown dead-zone input," Mechanical Systems and Signal Processing, vol. 111, pp. 135-148, 2018.

[10] L. Liu, X. S. Li, Y. J. Liu, and S. C. Tong, "Neural network based adaptive event trigger control for a class of electromagnetic suspension systems," Control Engineering Practice, vol. 106, Article ID 104675, 2021.

[11] F. León-Vargas, F. Garelli, and M. Zapateiro, "Limiting vertical acceleration for ride comfort in active suspension systems," Proceedings of the Institution of Mechanical 
Engineers, Part I: Journal of Systems and Control Engineering, vol. 232, no. 3, pp. 223-232, 2017.

[12] Y. Huang, J. Na, X. Wu, G.-B. Gao, and Y. Guo, "Robust adaptive control for vehicle active suspension systems with uncertain dynamics," Transactions of the Institute of Measurement and Control, vol. 40, no. 4, pp. 1237-1249, 2018.

[13] J. Na, Y. Huang, X. Wu, G. Gao, H. Guido, and Z. J. Jason, "Active adaptive estimation and control for vehicle suspensions with prescribed performance," IEEE Transactions on Control Systems Technology, vol. 26, no. 6, pp. 2063-2077, 2017.

[14] W. Gao, N. Zhang, and H. P. Du, "A half-car model for dynamic analysis of vehicles with random parameters," Proceedings of the 5th Australasian Congress on Applied Mechanics (ACAM 2007), Engineers Australia, vol. 1, pp. 595-600, 2007.

[15] H. Li, J. Yu, C. Hilton, and H. Liu, “Adaptive sliding-mode control for nonlinear active suspension vehicle systems using T-S fuzzy approach," IEEE Transactions on Industrial Electronics, vol. 58, no. 8, pp. 3328-3338, 2012.

[16] H. Li, H. Liu, C. Hilton, and S. Hand, "Non-fragile Ho control for half-vehicle active suspension systems with actuator uncertainties," Journal of Vibration and Control, vol. 19, no. 4, pp. 560-575, 2013.

[17] S. Sui, C. L. P. Chen, and S. Tong, "A novel adaptive NN prescribed performance control for stochastic nonlinear systems," IEEE Transactions on Neural Networks and Learning Systems, pp. 1-10, 2020.

[18] D.-P. Li, D.-J. Li, Y.-J. Liu, S. Tong, and C. L. P. Chen, "Approximation-based adaptive neural tracking control of nonlinear MIMO unknown time-varying delay systems with full state constraints," IEEE Transactions on Cybernetics, vol. 47, no. 10, pp. 3100-3109, 2017.

[19] W. He, Y. Dong, and C. Sun, "Adaptive neural network control of unknown nonlinear affine systems with input dead zone and output constraint," ISA Transactions, vol. 58, pp. 96-104, 2015

[20] Z. Liu, G. Lai, Y. Zhang, and C. L. P. Chen, “Adaptive fuzzy tracking control of nonlinear time-delay systems with deadzone output mechanism based on a novel smooth model," IEEE Transactions on Fuzzy Systems, vol. 23, no. 6, pp. 1998-2011, 2015.

[21] L. Liu, T. Gao, Y.-J. Liu, S. Tong, C. L. P. Chen, and L. Ma, "Time-varying IBLFs-based adaptive control of uncertain nonlinear systems with full state constraints," Automatica, vol. 129, Article ID 109595, 2021.

[22] W. He, Z. Yin, and C. Sun, "Adaptive neural network control of a marine vessel with constraints using the asymmetric barrier Lyapunov function," IEEE Transactions on Cybernetics, vol. 47, no. 7, pp. 1641-1651, 2017.

[23] Y. J. Liu, S. C. Tong, C. L. P. Chen, and D. J. Li, “Adaptive NN control using integral barrier Lyapunov functionals for uncertain nonlinear block-triangular constraint systems," IEEE Transactions on Cybernetics, vol. 47, no. 11, pp. 3747-3757, 2016.

[24] W. He, X. Mu, Y. Chen, X. He, and Y. Yu, "Modeling and vibration control of the flapping-wing robotic aircraft with output constraint," Journal of Sound and Vibration, vol. 423, pp. 472-483, 2018.

[25] W. Sun, H. Pan, Y. Zhang, and H. Gao, "Multi-objective control for uncertain nonlinear active suspension systems," Mechatronics, vol. 24, no. 4, pp. 318-327, 2013.

[26] Y.-J. Liu, Q. Zeng, S. Tong, C. L. P. Chen, and L. Liu, "Adaptive neural network control for active suspension systems with time-varying vertical displacement and speed constraints," IEEE Transactions on Industrial Electronics, vol. 66, no. 12, pp. 9458-9466, 2019.

[27] S. P. Bhat and D. S. Bernstein, "Continuous finite-time stabilization of the translational and rotational double integrators," IEEE Transactions on Automatic Control, vol. 43, no. 5, pp. 678-682, 2018.

[28] P. Zhao, D.-H. Zhai, Y. Sun, and Y. Li, "Adaptive finite-time control of a class of Markovian jump nonlinear systems with parametric and dynamic uncertainties," Nonlinear Analysis: Hybrid Systems, vol. 29, pp. 234-246, 2018.

[29] S. Sui, C. L. P. Chen, and S. Tong, "Event-trigger-based finitetime fuzzy adaptive control for stochastic nonlinear system with unmodeled dynamics," IEEE Transactions on Fuzzy Systems, vol. 29, 2020.

[30] R. Li and J. Cao, "Finite-time stability analysis for Markovian jump memristive neural networks with partly unknown transition probabilities," IEEE Transactions on Neural Networks and Learning Systems, vol. 28, no. 12, pp. 2924-2935, 2018.

[31] Y. Hong, J. Wang, and D. Cheng, "Adaptive finite-time control of nonlinear systems with parametric uncertainty," IEEE Transactions on Automatic Control, vol. 51, no. 5, pp. 858-862, 2005.

[32] Y. Dan, X. D. Li, and J. L. Qiu, "Output tracking control of delayed switched systems via state-dependent switching and dynamic output feedback," Nonlinear Analysis: Hybrid Systems, vol. 32, pp. 294-305, 2019.

[33] D. Yang, X. Li, J. Shen, and Z. Zhou, "State-dependent switching control of delayed switched systems with stable and unstable modes," Mathematical Methods in the Applied Sciences, vol. 41, no. 16, pp. 6968-6983, 2018.

[34] S. Sui, C. L. P. Chen, and S. Tong, "Neural-network-based adaptive DSC design for switched fractional-order nonlinear systems," IEEE Transactions on Neural Networks and Learning Systems, pp. 1-10, 2020.

[35] X. Zhang, G. Feng, and Y. Sun, "Finite-time stabilization by state feedback control for a class of time-varying nonlinear systems," Automatica, vol. 48, no. 3, pp. 499-504, 2012.

[36] H. Wang, B. Chen, C. Lin, Y. Sun, and F. Wang, "Adaptive finite-time control for a class of uncertain high-order non-linear systems based on fuzzy approximation," IET Control Theory \& Applications, vol. 11, no. 5, pp. 677-684, 2017.

[37] M. Cai and Z. Xiang, "Adaptive practical finite-time stabilization for uncertain nonstrict feedback nonlinear systems with input nonlinearity," IEEE Transactions on Systems, Man, and Cybernetics: Systems, vol. 47, no. 7, pp. 1668-1678, 2017.

[38] S. Sui, C. L. P. Chen, and S. C. Tong, "Fuzzy adaptive finitetime control design for nontriangular stochastic nonlinear systems," IEEE Transactions on Fuzzy Systems, vol. 27, no. 1, pp. 172-184, 2018.

[39] H. Pan, W. Sun, H. Gao, and J. Yu, "Finite-time stabilization for vehicle active suspension systems with hard constraints," IEEE Transactions on Intelligent Transportation Systems, vol. 16, no. 5, pp. 2663-2672, 2015.

[40] G. Wang, C. Chen, and S. Yu, "Finite-time sliding mode tracking control for active suspension systems via extended super-twisting observer," Proceedings of the Institution of Mechanical Engineers, Part I: Journal of Systems and Control Engineering, vol. 231, no. 6, pp. 459-470, 2017.

[41] Y. J. Liu, Y. Zhang, L. Liu, S. C. Tong, and C. L. P. Chen, "Adaptive finite-time control for half-vehicle active suspension systems with uncertain dynamics," IEEE/ASME Transactions on Mechatronics, vol. 26, no. 1, pp. 168-178, 2020. 
[42] S. Zhao, H. Liang, P. Du, and S. Qi, “Adaptive NN finite-time tracking control of output constrained nonlinear system with input saturation," Nonlinear Dynamics, vol. 92, no. 4, pp. 1845-1856, 2018.

[43] R. Ma, Y. Liu, S. Zhao, and J. Fu, "Finite-time stabilization of a class of output-constrained nonlinear systems," Journal of the Franklin Institute, vol. 352, no. 12, pp. 5968-5984, 2015.

[44] Z. Gao and P. Antsaklis, "Stability of the pseudo-inverse method for reconfigurable control systems," International Journal of Control, vol. 53, no. 3, pp. 717-729, 1996.

[45] M. M. Kale and A. J. Chipperfield, "Stabilized MPC formulations for robust reconfigurable flight control," Control Engineering Practice, vol. 13, no. 6, pp. 771-788, 2005.

[46] M. L. Corradini and G. Orlando, "Actuator failure identification and compensation through sliding modes," IEEE Transactions on Control Systems Technology, vol. 15, no. 1, pp. 184-190, 2006.

[47] G. Tao, S. M. Joshi, and X. Ma, "Adaptive state feedback and tracking control of systems with actuator failures," IEEE Transactions on Automatic Control, vol. 46, no. 1, pp. 78-95, 2001.

[48] G. Lai, C. Wen, Z. Liu, Y. Zhang, and S. Xie, "Adaptive compensation for infinite number of actuator failures/faults using output feedback control," Information Sciences, vol. 399, pp. 1-12, 2017.

[49] L. Liu, Y.-J. Liu, and S. Tong, "Neural networks-based adaptive finite-time fault-tolerant control for a class of strictfeedback switched nonlinear systems," IEEE Transactions on Cybernetics, vol. 49, no. 7, pp. 2536-2545, 2019.

[50] Y.-J. Liu, S. Li, S. Tong, and C. L. P. Chen, "Neural approximation-based adaptive control for a class of nonlinear nonstrict feedback discrete-time systems," IEEE Transactions on Neural Networks and Learning Systems, vol. 28, no. 7, pp. 1531-1541, 2017.

[51] O. L. V. Costa, M. D. Fragoso, and R. P. Marques, Discrete Time Markov Jump Linear Systems, Springer-Verlag, New York, NY, USA, 2005.

[52] H. Fan, B. Liu, Y. Shen, and W. Wang, "Adaptive failure compensation control for uncertain systems with stochastic actuator failures," IEEE Transactions on Automatic Control, vol. 59, no. 3, pp. 808-814, 2014.

[53] B. Liu, M. Saif, and H. Fan, "Adaptive fault tolerant control of a half-car active suspension systems subject to random actuator failures," IEEE/ASME Transactions on Mechatronics, vol. 21, no. 6, pp. 2847-2857, 2016.

[54] J. Yin, S. Khoo, Z. Man, and X. Yu, "Finite-time stability and instability of stochastic nonlinear systems," Automatica, vol. 47, no. 12, pp. 2671-2677, 2011.

[55] S. Khoo, J. Yin, Z. Man, and X. Yu, "Finite-time stabilization of stochastic nonlinear systems in strict-feedback form," Automatica, vol. 49, no. 5, pp. 1403-1410, 2013.

[56] R. Wang, Q. Sun, W. Hu, Y. Li, D. Ma, and P. Wang, "SoCbased droop coefficients stability region analysis of the battery for stand-alone supply systems with constant power loads," IEEE Transactions on Power Electronics, vol. 36, no. 7, pp. 7866-7879, 2021.

[57] Q. Sun, R. Han, H. Zhang, J. Zhou, and J. M. Guerrero, “A multiagent-based consensus algorithm for distributed coordinated control of distributed generators in the energy internet," IEEE Transactions on Smart Grid, vol. 6, no. 6, pp. 3006-3019, 2015.

[58] X. Hu, H. Zhang, D. Ma, and R. Wang, "Hierarchical pressure data recovery for pipeline network via generative adversarial networks," IEEE Transactions on Automation Science and Engineering, pp. 1-11, 2021.

[59] D. Ma, Y. Li, X. Hu, H. Zhang, and X. Xie, "An optimal threedimensional drone layout method for maximum signal coverage and minimum interference in complex pipeline networks," IEEE Transactions on Cybernetics, pp. 1-11, 2021. 